\title{
Entropy Solutions for Nonlinear Elliptic Anisotropic Homogeneous Neumann Problem
}

\author{
B. K. Bonzi, S. Ouaro, and F. D. Y. Zongo \\ Laboratoire d'Analyse Mathématique des Equations (LAME), UFR, Sciences Exactes et Appliquées, Université de Ouagadougou, \\ 03 BP 7021 Ouaga 03, Ouagadougou, Burkina Faso
}

Correspondence should be addressed to S. Ouaro; ouaro@yahoo.fr

Received 22 June 2012; Accepted 1 February 2013

Academic Editor: Elena Braverman

Copyright ( 92013 B. K. Bonzi et al. This is an open access article distributed under the Creative Commons Attribution License, which permits unrestricted use, distribution, and reproduction in any medium, provided the original work is properly cited.

We prove the existence and uniqueness of entropy solution for nonlinear anisotropic elliptic equations with Neumann homogeneous boundary value condition for $L^{1}$-data. We prove first, by using minimization techniques, the existence and uniqueness of weak solution when the data is bounded, and by approximation methods, we prove a result of existence and uniqueness of entropy solution.

\section{Introduction}

Let $\Omega$ be an open bounded domain of $\mathbb{R}^{N}(N \geq 3)$ with smooth boundary. Our aim is to prove the existence and uniqueness of entropy solution for the anisotropic nonlinear elliptic problem of the form

$$
\begin{gathered}
-\sum_{i=1}^{N} \frac{\partial}{\partial x_{i}} a_{i}\left(x, \frac{\partial}{\partial x_{i}} u\right)+|u|^{p_{M}(x)-2} u=f \quad \text { in } \Omega, \\
\sum_{i=1}^{N} a_{i}\left(x, \frac{\partial}{\partial x_{i}} u\right) v_{i}=0 \quad \text { on } \partial \Omega,
\end{gathered}
$$

where the right-hand side $f \in L^{1}(\Omega)$ and $v_{i}, i \in\{1, \ldots, N\}$ are the components of the outer normal unit vector.

For the rest of the functions involved in (1), we are going to enumerate their properties after we make some notations.

For any $\Omega \subset \mathbb{R}^{N}$, we set

$$
C_{+}(\bar{\Omega})=\left\{h \in C(\bar{\Omega}): \inf _{x \in \Omega} h(x)>1\right\},
$$

and we denote

$$
h^{+}=\sup _{x \in \Omega} h(x), \quad h^{-}=\inf _{x \in \Omega} h(x) .
$$

For the exponents, $\vec{p}(\cdot): \bar{\Omega} \rightarrow \mathbb{R}^{N}, \vec{p}(\cdot)=\left(p_{1}(\cdot), \ldots, p_{N}(\cdot)\right)$ with $p_{i} \in C_{+}(\bar{\Omega})$ for every $i \in\{1, \ldots, N\}$ and for all $x \in$
$\bar{\Omega}$, we put $p_{M}(x)=\max \left\{p_{1}(x), \ldots, p_{N}(x)\right\}$ and $p_{m}(x)=$ $\min \left\{p_{1}(x), \ldots, p_{N}(x)\right\}$. Now, we can give the properties of the rest of the functions involved in (1).

We assume that for $i=1, \ldots, N$, the function $a_{i}$ : $\Omega \times \mathbb{R} \rightarrow \mathbb{R}$ is Carathéodory and satisfies the following conditions: $a_{i}(x, \xi)$ is the continuous derivative with respect to $\xi$ of the mapping $A_{i}: \Omega \times \mathbb{R} \rightarrow \mathbb{R}, A_{i}=A_{i}(x, \xi)$, that is, $a_{i}(x, \xi)=(\partial / \partial \xi) A_{i}(x, \xi)$ such that the following equality and inequalities holds

$$
A_{i}(x, 0)=0,
$$

for almost every $x \in \Omega$.

There exists a positive constant $C_{1}$ such that

$$
\left|a_{i}(x, \xi)\right| \leq C_{1}\left(j_{i}(x)+|\xi|^{p_{i}(x)-1}\right),
$$

for almost every $x \in \Omega$ and for every $\xi \in \mathbb{R}$, where $j_{i}$ is a nonnegative function in $L^{p_{i}^{\prime}(\cdot)}(\Omega)$, with $1 / p_{i}(x)+1 / p_{i}^{\prime}(x)=1$.

There exists a positive constant $C_{2}$ such that

$$
\begin{aligned}
& \left(a_{i}(x, \xi)-a_{i}(x, \eta)\right) \cdot(\xi-\eta) \\
& \quad \geq \begin{cases}C_{2}|\xi-\eta|^{p_{i}(x)} & \text { if }|\xi-\eta| \geq 1, \\
C_{2}|\xi-\eta|^{p_{i}^{-}} & \text {if }|\xi-\eta|<1,\end{cases}
\end{aligned}
$$


for almost every $x \in \Omega$ and for every $\xi, \eta \in \mathbb{R}$, with $\xi \neq \eta$ and

$$
|\xi|^{p_{i}(x)} \leq a_{i}(x, \xi) \cdot \xi \leq p_{i}(x) A_{i}(x, \xi),
$$

for almost every $x \in \Omega$ and for every $\xi \in \mathbb{R}$.

We also assume that the variable exponents $p_{i}(\cdot): \bar{\Omega} \rightarrow$ $[2, N)$ are continuous functions for all $i=1, \ldots, N$ such that

$$
\begin{gathered}
\frac{\bar{p}(N-1)}{N(\bar{p}-1)}<p_{i}^{-}<\frac{\bar{p}(N-1)}{N-\bar{p}}, \quad \sum_{i=1}^{N} \frac{1}{p_{i}^{-}}>1, \\
\frac{p_{i}^{+}-p_{i}^{-}-1}{p_{i}^{-}}<\frac{\bar{p}-N}{\bar{p}(N-1)},
\end{gathered}
$$

where $1 / \bar{p}=(1 / N) \sum_{i=1}^{N}\left(1 / p_{i}^{-}\right)$.

We introduce the numbers

$$
q=\frac{N(\bar{p}-1)}{N-1}, \quad q^{*}=\frac{N q}{N-q}=\frac{N(\bar{p}-1)}{N-\bar{p}} .
$$

A prototype example, that is, covered by our assumptions is the following anisotropic equation:

Set $A_{i}(x, \xi)=\left(1 / p_{i}(x)\right)|\xi|^{p_{i}(x)}, a_{i}(x, \xi)=|\xi|^{p_{i}(x)-2} \xi$ where $p_{i}(x) \geq 2$. Then, we get the following equation.

$$
-\sum_{i=1}^{N} \frac{\partial}{\partial x_{i}}\left(\left|\frac{\partial}{\partial x_{i}} u\right|^{p_{i}(x)-2} \frac{\partial}{\partial x_{i}} u\right)+|u|^{p_{M}(x)-2}=f .
$$

Actually, one of the topics from the field of PDEs that continuously gained interest is the one concerning the Sobolev space with variable exponents, $W^{1, p(\cdot)}(\Omega)$ or $W_{0}^{1, p(\cdot)}(\Omega)$ depending on the boundary condition (see [1-23]). In that context, problems involving the $p(\cdot)$-Laplace operator

$$
\Delta_{p(x)} u=\operatorname{div}\left(|\nabla u|^{p(x)-2} \nabla u\right)
$$

or the more general operator

$$
\operatorname{div} a(x, \nabla u)
$$

were intensively studied (see [13]). At the same time, some authors was interested by PDEs involving anisotropic Sobolev spaces with variable exponent $W^{1, \vec{p}(\cdot)}$ when the boundary condition is the homogeneous Dirichlet boundary condition (see $[15,16,18,20,24-26])$. In that context, the authors have considered the anisotropic $p(\cdot)$-Laplace operator

$$
\Delta_{\vec{p}(x)} u=\sum_{i=1}^{N} \partial_{x_{i}}\left(\left|\partial_{x_{i}} u\right|^{p_{i}(x)-2} \partial_{x_{i}} u\right)
$$

or the more general variable exponent anisotropic operator

$$
\sum_{i=1}^{N} \partial_{x_{i}} a\left(x, \partial_{x_{i}} u\right) \text {. }
$$

When the homogeneous Dirichlet boundary condition is replaced by the Neumann boundary condition, one has to work with the anisotropic variable exponent Sobolev space
$W^{1, \vec{p}^{(\cdot)}}(\Omega)$ instead of $W_{0}^{1, \vec{p}(\cdot)}(\Omega)$. The main difficulty which appears is that the famous Poincaré inequality does not apply and then it is very difficult to get a priori estimates which are necessary for the proof of the existence result of entropy solutions. Sometimes one can use the Wirtinger inequality which does not apply, in some problems like (1). The first systematic study of anisotropic Neumann problem was done by Fan (see [11]). In a second time, Boureanu and Rădulescu studied an anisotropic nonhomogeneous Neumann problem with obstacle (see [2]). In the two papers, the authors were interested by the existence and multiplicity results of weak solution even if in [2], Boureanu and Rădulescu have showed some conditions under which we can get uniqueness of weak solution. In this paper, we are interested to the existence and uniqueness of entropy solution. For the proof of the existence of entropy solution of (1), we follow [27] and derive a priori estimates for the approximated solutions $u_{n}$ and the partial derivatives $\partial u_{n} / \partial x_{i}$ in the Marcinkiewicz spaces $\mathscr{M}^{\widetilde{p}}$ and $\mathscr{M}^{\tilde{p}_{i}}$, respectively (see Section 2 or $[27,28]$ for definition and properties of Marcinkiewicz spaces).

The study of anisotropic problems are motivated, for example, by their applications to the mathematical analysis of a system of nonlinear partial differential equations arising in a population dynamics model describing the spread of an epidemic disease through a heterogeneous habitat.

The paper is organized as follows. In Section 2, we introduce some notations/functional spaces. In Section 3, we prove for the problem (1), the existence and uniqueness of weak solution when the data is bounded, and the existence and uniqueness of entropy solution when the data is in $L^{1}(\Omega)$.

\section{Preliminaries}

In this section, we define Lebesgue, Sobolev, and anisotropic spaces with variable exponent and give some of their properties (see [29] for more details about Lebesgue and Sobolev spaces with variable exponent). Roughly speaking, anistropic Lebesgue and Sobolev spaces are functional spaces of Lebesgue's and Sobolev's type in which different space directions have different roles.

Given a measurable function $p(\cdot): \Omega \rightarrow[1, \infty)$, we define the Lebesgue space with variable exponent $L^{p(\cdot)}(\Omega)$ as the set of all measurable functions $u: \Omega \rightarrow \mathbb{R}$ for which the convex modular

$$
\rho_{p(\cdot)}(u):=\int_{\Omega}|u|^{p(x)} d x
$$

is finite. If the exponent is bounded, that is, if $p_{+}<\infty$, then the expression

$$
|u|_{p(\cdot)}:=\inf \left\{\lambda>0: \rho_{p(\cdot)}\left(\frac{u}{\lambda}\right) \leq 1\right\}
$$

defines a norm in $L^{p(\cdot)}(\Omega)$, called the Luxembourg norm. The space $\left(L^{p(\cdot)}(\Omega),|\cdot|_{p(\cdot)}\right)$ is a separable Banach space. Moreover, if $p_{-}>1$, then $L^{p(\cdot)}(\Omega)$ is uniformly convex, hence reflexive, and its dual space is isomorphic to $L^{p^{\prime}(\cdot)}(\Omega)$, where $1 / p(x)+$ $1 / p^{\prime}(x)=1$. Finally, we have the Hölder type inequality. 
Proposition 1 (generalized Hölder inequality, see [10]). (i) For any $u \in L^{p(\cdot)}(\Omega)$ and $v \in L^{p^{(\cdot)}(\Omega) \text {, we have }}$

$$
\left|\int_{\Omega} u v d x\right| \leq\left(\frac{1}{p_{-}}+\frac{1}{p_{-}^{\prime}}\right)|u|_{p(\cdot)}|v|_{p^{\prime}(\cdot)} .
$$

(ii) If $p_{1}, p_{2} \in \mathscr{C}_{+}(\bar{\Omega}), p_{1}(x) \leq p_{2}(x)$ for any $x \in \bar{\Omega}$, then $L^{p_{2}(x)}(\Omega) \hookrightarrow L^{p_{1}(x)}(\Omega)$ and the imbedding is continuous.

Moreover, the application $\rho_{p(\cdot)}: L^{p(\cdot)}(\Omega) \rightarrow \mathbb{R}$ called the $p(\cdot)$-modular of the $L^{p(\cdot)}(\Omega)$ space is very useful in handling these Lebesgue spaces with variable exponent. Indeed we have the following properties (see [10]). If $u \in L^{p(\cdot)}(\Omega)$ and $p<\infty$ then

$$
\begin{gathered}
|u|_{p(\cdot)}<1 \Longrightarrow|u|_{p(\cdot)}^{p^{+}} \leq \rho_{p(\cdot)}(u) \leq|u|_{p(\cdot)}^{p^{-}}, \\
|u|_{p(\cdot)}>1 \Longrightarrow|u|_{p(\cdot)}^{p^{-}} \leq \rho_{p(\cdot)}(u) \leq|u|_{p(\cdot)}^{p^{+}}, \\
|u|_{p(\cdot)}<1(=1 ;>1) \Longrightarrow \rho_{p(\cdot)}(u)<1(=1 ;>1), \\
|u|_{p(\cdot)} \longrightarrow 0(\longrightarrow \infty) \Longleftrightarrow \rho_{p(\cdot)}(u) \longrightarrow 0(\longrightarrow \infty) .
\end{gathered}
$$

If, in addition, $\left(u_{n}\right)_{n} \subset L^{p(\cdot)}(\Omega)$, then

$\lim _{n \rightarrow \infty}\left|u_{n}-u\right|_{p(\cdot)}=0 \Leftrightarrow \lim _{n \rightarrow \infty} \rho_{p(\cdot)}\left(u_{n}-u\right)=$ $0 \Leftrightarrow\left(u_{n}\right)_{n}$ converges to $u$ in measure and $\lim _{n \rightarrow \infty} \rho_{p(\cdot)}\left(u_{n}\right)=$ $\rho_{p(\cdot)}(u)$.

Now, let us introduce the definition of the isotropic Sobolev space with variable exponent, $W^{1, p(\cdot)}(\Omega)$.

We set

$$
W^{1, p(\cdot)}(\Omega):=\left\{u \in L^{p(\cdot)}(\Omega):|\nabla u| \in L^{p(\cdot)}(\Omega)\right\},
$$

which is a Banach space equipped with the norm

$$
\|u\|_{1, p(\cdot)}:=|u|_{p(\cdot)}+|\nabla u|_{p(\cdot)} .
$$

Now, we present a natural generalization of the variable exponent Sobolev space $W^{1, p(\cdot)}(\Omega)$ that will enable us to study the problem (1) with sufficient accuracy.

The anisotropic variable exponent Sobolev space $W^{1, \vec{p}(\cdot)}(\Omega)$ is defined as follows:

$$
\begin{aligned}
& W^{1, \vec{p}(\cdot)}(\Omega) \\
& \quad=\left\{u \in L^{p_{M}(\cdot)}(\Omega) ; \frac{\partial u}{\partial x_{i}} \in L^{p_{i}(\cdot)}(\Omega), \quad \forall i \in\{1, \ldots, N\}\right\} .
\end{aligned}
$$

Endowed with the norm

$$
\|u\|_{\vec{p}(\cdot)}:=|u|_{p_{M}(\cdot)}+\sum_{i=1}^{N}\left|\frac{\partial}{\partial x_{i}} u\right|_{p_{i}(\cdot)},
$$

the space $\left(W^{1, \vec{p}(\cdot)}(\Omega),\|\cdot\|_{\vec{p}(\cdot)}\right)$ is a reflexive Banach space (see [11, Theorems 2.1 and 2.2]).

We have the following result.
Theorem 2 (see [11, Corollary 2.1]). Let $\Omega \subset \mathbb{R}^{N}(N \geq 3)$ be a bounded open set and for all $i \in\{1, \ldots, N\}, p_{i} \in L^{\infty}(\Omega)$, $p_{i}(x) \geq 1$ a.e. in $\Omega$. Then, for any $r \in L^{\infty}(\Omega)$ with $r(x) \geq 1$ a.e. in $\Omega$ such that

$$
\text { ess } \inf _{x \in \Omega}\left(p_{M}(x)-r(x)\right)>0,
$$

we have the compact embedding

$$
W^{1, \vec{p}(\cdot)}(\Omega) \hookrightarrow L^{r(\cdot)}(\Omega) .
$$

Next, we define

$$
\mathscr{T}^{1, \vec{p}^{(\cdot)}}(\Omega)=\left\{u: \Omega \rightarrow \mathbb{R} ; T_{k}(u) \in W^{1, \vec{p}^{(\cdot)}}(\Omega), \forall k>0\right\} .
$$

Finally, in this paper, we will use the Marcinkiewicz spaces $\mathscr{M}^{q}(\Omega)(1<q<\infty)$ with constant exponent. Note that the Marcinkiewicz spaces $\mathscr{M}^{q(\cdot)}(\Omega)$ in the variable exponent setting were introduced for the first time by Sanchon and Urbano (see [23]).

Marcinkiewicz spaces $\mathscr{M}^{q}(\Omega)(1<q<\infty)$ contain the measurable functions $h: \Omega \rightarrow \mathbb{R}$ for which the distribution function

$$
\lambda_{h}(\gamma)=|\{x \in \Omega:|h(x)|>\gamma\}|, \quad \gamma \geq 0
$$

satisfies an estimate of the form

$$
\lambda_{h}(\gamma) \leq C \gamma^{-q}, \quad \text { for some finite constant } C>0 .
$$

The space $\mathscr{M}^{q}(\Omega)$ is a Banach space under the norm

$$
\|h\|_{\mathscr{M}^{q}(\Omega)}^{*}=\sup _{t>0} t^{1 / q}\left(\frac{1}{t} \int_{0}^{t} h^{*}(s) d s\right),
$$

where $h^{*}$ denotes the nonincreasing rearrangement of $h$ :

$$
h^{*}(t)=\inf \left\{\gamma>0: \lambda_{h}(\gamma) \leq t\right\} .
$$

We will use the following pseudonorm

$$
\|h\|_{\mathscr{M}^{q}(\Omega)}=\inf \left\{C: \lambda_{h}(\gamma) \leq C \gamma^{-q}, \forall \gamma>0\right\},
$$

which is equivalent to the norm $\|h\|_{\mathscr{M}^{q}(\Omega)}^{*}$ (see [27]).

We need the following Lemma (see [28, Lemma A.2]).

Lemma 3. Let $1 \leq q<p<+\infty$. Then, for every measurable function $u$ on $\Omega$, we have

(i) $\left((p-1)^{p} / p^{p+1}\right)\|u\|_{\mathscr{M}^{p}(\Omega)}^{p} \leq \sup _{\lambda>0}\left\{\lambda^{p} \operatorname{meas}[x \in \Omega\right.$ : $|u(x)|>\lambda]\} \leq\|u\|_{\mathscr{M}^{p}(\Omega)}^{p}$.

Moreover,

(ii) $\int_{K}|u|^{q} d x \leq(p /(p-q))(p / q)^{q / p}\|u\|_{\mathcal{M}^{p}(\Omega)}^{q}$ $(\text { meas }(K))^{p-q / p}$, for every measurable subset $K \subset \Omega$.

In particular, $\mathscr{M}^{p}(\Omega) \subset L_{\text {loc }}^{q}(\Omega)$ with continuous injection and $u \in \mathscr{M}^{p}(\Omega)$ implies $|u|^{q} \in \mathscr{M}^{p / q}(\Omega)$. 
The following result is due to Troisi (see [30]).

Theorem 4. Let $p_{1}, p_{2}, \ldots, p_{N} \in[1,+\infty) ; g \in W^{1,\left(p_{1}, p_{2}, \ldots, p_{N}\right)}$ $(\Omega)$ and let

$$
\begin{gathered}
q=\bar{p}^{*} \quad \text { if } \bar{p}^{*}<N, \\
q \in[1,+\infty) \quad \text { if } \bar{p}^{*} \geq N .
\end{gathered}
$$

Then, there exists a constant $C>0$ depending on $N, p_{1}, p_{2}, \ldots, p_{N}$ if $\bar{p}<N$ and also on $q$ and meas $(\Omega)$ if $\bar{p} \geq N$ such that

$$
\|g\|_{L^{q}(\Omega)} \leq C \prod_{i=1}^{N}\left[\|g\|_{L^{p_{M}(\Omega)}}+\left\|\frac{\partial g}{\partial x_{i}}\right\|_{L^{p_{i}}(\Omega)}\right]^{1 / N}
$$

where $p_{M}=\max \left\{p_{1}, p_{2}, \ldots, p_{N}\right\}$ and $1 / \bar{p}=(1 / N)$ $\sum_{i=1}^{N}\left(1 / p_{i}\right)$.

We will use through the paper, the truncation function $T_{\gamma}$ at height $(\gamma>0)$, that is

$$
T_{\gamma}(s)= \begin{cases}s & \text { if }|s| \leq \gamma \\ \gamma \operatorname{sign}(s) & \text { if }|s|>\gamma .\end{cases}
$$

We need the following lemma.

Lemma 5. Let $g$ be a nonnegative function in $W^{1, \vec{p}(\cdot)}(\Omega)$. Assume $\bar{p}<N$ and there exists a constant $C>0$ such that

$$
\begin{gathered}
\int_{\Omega}\left|T_{\gamma}(g)\right|^{p_{M}^{-}} d x+\sum_{i=1}^{N} \int_{\{|g| \leq \gamma\}}\left|\frac{\partial g}{\partial x_{i}}\right|^{p_{i}^{-}} d x \\
\leq C(\gamma+1), \quad \forall \gamma>0 .
\end{gathered}
$$

Then, there exists a constant $D$, depending on $C$, such that

$$
\|g\|_{\mu^{\tilde{p}}(\Omega)} \leq D,
$$

where $\widetilde{p}=N(\bar{p}-1) /(N-\bar{p})$.

Proof. Consider the following

Step $1\left(\left\|T_{\gamma}(g)\right\|_{L^{p_{M}^{-}(\Omega)}} \leq 1\right)$. Then, obviously we have $\|g\|_{\mu^{\tilde{P}}(\Omega)} \leq D$, for some positive constant $D$. Indeed, since $1<\widetilde{p} \leq \bar{p} \leq p_{M}^{-}$, according to Proposition 1 there exists a positive constant $C$ such that

$$
\left\|T_{\gamma}(g)\right\|_{L^{\tilde{p}}(\Omega)} \leq C\left\|T_{\gamma}(g)\right\|_{L^{p_{M}^{-}(\Omega)}} \leq C .
$$

It follows that there exists a positive constant $D$ such that

$$
\|g\|_{\mu^{\tilde{p}}(\Omega)} \leq D .
$$

Step $2\left(\left\|T_{\gamma}(g)\right\|_{L^{p_{M}^{-}(\Omega)}}>1\right)$. We get from (37)

$$
\left\|T_{\gamma}(g)\right\|_{L^{p_{M}^{-}(\Omega)}}^{p_{M}^{-}}+\left\|\frac{\partial T_{\gamma}(g)}{\partial x_{i}}\right\|_{L^{p_{i}^{-}(\Omega)}}^{p_{i}^{-}} \leq C(\gamma+1) .
$$

Not also that

$$
\begin{aligned}
& \left(\left\|T_{\gamma}(g)\right\|_{L^{p_{M}^{-}(\Omega)}}+\left\|\frac{\partial T_{\gamma}(g)}{\partial x_{i}}\right\|_{L^{p_{i}^{-}}(\Omega)}\right)^{p_{i}^{-}} \\
& \quad \leq 2^{\left(p_{i}^{-}-1\right)}\left(\left\|T_{\gamma}(g)\right\|_{L^{p_{M}^{-}(\Omega)}}^{p^{-}}+\left\|\frac{\partial T_{\gamma}(g)}{\partial x_{i}}\right\|_{L^{p_{i}^{-}(\Omega)}}^{p_{i}^{-}}\right) \\
& \quad \leq 2^{\left(p_{i}^{-}-1\right)}\left(\left\|T_{\gamma}(g)\right\|_{L^{p_{M}^{-}(\Omega)}}^{p^{-}}+\left\|\frac{\partial T_{\gamma}(g)}{\partial x_{i}}\right\|_{L^{p_{i}^{-}(\Omega)}}^{p_{i}^{-}}\right) .
\end{aligned}
$$

Therefore, by using (35), we obtain for $\gamma>1$,

$$
\begin{aligned}
\left\|T_{\gamma}(g)\right\|_{L^{q}(\Omega)} & \leq C \prod_{i=1}^{N}\left[2^{\left(p_{i}^{-}-1\right) / N p_{i}^{-}} \gamma^{1 / N p_{i}^{-}}\right] \\
& \leq D \gamma^{\sum_{i=1}^{N}\left(1 / N p_{i}^{-}\right)}=D \gamma^{1 / \bar{p}} .
\end{aligned}
$$

It follows that

$$
\int_{\{|g|>\gamma\}}\left|T_{\gamma}(g)\right|^{q} d x \leq D \gamma^{q / \bar{p}}
$$

which is equivalent to

$$
\gamma^{q} \text { meas }(\{|g|>\gamma\}) \leq D \gamma^{q / \bar{p}}
$$

Therefore,

$$
\operatorname{meas}(\{|g|>\gamma\}) \leq D \gamma^{-q(\bar{p}-1) / \bar{p}} .
$$

Since, $q=\bar{p}^{*}=N \bar{p} /(N-\bar{p})$ we get

$$
\operatorname{meas}(\{|g|>\gamma\}) \leq D \gamma^{-N(\bar{p}-1) /(N-\bar{p})}
$$

which implies that $\|g\|_{\mathscr{M} \tilde{p}(\Omega)} \leq D$.

For $0<\gamma \leq 1$ we have

$$
\operatorname{meas}(\{|g|>\gamma\}) \leq \operatorname{meas}(\Omega) \leq \operatorname{meas}(\Omega) \gamma^{-\widetilde{p}} \text {. }
$$

So,

$$
\|g\|_{\mu^{\tilde{p}}(\Omega)} \leq D .
$$

We need the following well-known results.

Theorem 6 (see [31, Theorem 6.2.1]). Let $X$ be a reflexive Banach space and let $f: M \subset X \rightarrow \mathbb{R}$ be Gateaux differentiable over the closed set $M$. Then, the following are equivalent.

(i) $f$ is convex over $M$.

(ii) We have

$$
\begin{array}{r}
f(u)-f(v) \geq\left\langle f^{\prime}(v), u-v\right\rangle_{X^{*} \times X} \\
\forall u, v \in M,
\end{array}
$$

where $X^{*}$ denotes the dual of the space $X$. 
(iii) The first Gateaux derivative is monotone, that is,

$$
\begin{array}{r}
\left\langle f^{\prime}(u)-f^{\prime}(v), u-v\right\rangle_{X^{*} \times X} \geq 0 \\
\forall u, v \in M .
\end{array}
$$

(iv) The second Gateaux derivative of $f$ exists and it is positive, that is,

$$
\left\langle f^{\prime \prime}(u) \circ v, v\right\rangle_{X^{*} \times X} \geq 0 \quad \forall v \in M .
$$

Theorem 7 (see [32, Theorem 1.2]). Suppose $X$ is a reflexive Banach space with norm $\|\cdot\|_{X}$, and let $M \subset X$ be a weakly closed subset of $X$. Suppose $\Psi: M \subset X \rightarrow \mathbb{R} \cup\{\infty\}$ is coercive and (sequentially) weakly lower semicontinuous on $M$ with respect to $X$, that is, suppose the following conditions are fulfilled.

(i) $\Psi(u) \rightarrow \infty a s\|u\|_{X} \rightarrow \infty, u \in M$.

(ii) For any $u \in M$, any subsequence $\left(u_{m}\right)$ in $M$ such that $u_{m} \rightarrow u$ weakly in $X$ there holds

$$
\Psi(u) \leq \liminf _{m \rightarrow \infty} \Psi\left(u_{m}\right) .
$$

Then, $\Psi$ is bounded from below and attains its infinimun in $M$.

\section{Main Results}

In the sequel, we denote $W^{1, \vec{p}(\cdot)}(\Omega)=E$ and $\|\cdot\|_{W^{1, \vec{p}(\cdot)}(\Omega)}=$ $\|\cdot\|_{E}$.

3.1. Weak Solutions. Let us define first the notion of weak solution.

Definition 8 . Let $u: \Omega \rightarrow \mathbb{R}$ be a measurable function, we say that $u$ is a weak solution of problem (1) if $u$ belongs to $W^{1, \vec{p}(\cdot)}(\Omega)$ and satisfies the following equation:

$$
\begin{aligned}
& \int_{\Omega} \sum_{i=1}^{N} a_{i}\left(x, \frac{\partial u}{\partial x_{i}}\right) \frac{\partial v}{\partial x_{i}} d x \\
& +\int_{\Omega}|u|^{p_{M}(x)-2} u v d x-\int_{\Omega} f(x) v d x=0,
\end{aligned}
$$

for every $v \in W^{1, \vec{p}(\cdot)}(\Omega)$.

We associate to problem (1) the energy functional $I: E \rightarrow$ $\mathbb{R}$, defined by

$$
\begin{aligned}
I(u)= & \int_{\Omega} \sum_{i=1}^{N} A_{i}\left(x, \frac{\partial u}{\partial x_{i}}\right) d x \\
& +\int_{\Omega} \frac{1}{p_{M}(x)}|u|^{p_{M}(x)} d x-\int_{\Omega} f(x) u d x .
\end{aligned}
$$

To simplify our writing, we denote by $\Lambda: E \rightarrow \mathbb{R}$ the functional

$$
\Lambda(u)=\int_{\Omega} \sum_{i=1}^{N} A_{i}\left(x, \frac{\partial u}{\partial x_{i}}\right) d x .
$$

We recall the following result (see $[15$, Lemma 3.4]).
Lemma 9. The functional $\Lambda$ is well-defined on $E$. In addition, $\Lambda$ is of class $\mathscr{C}^{1}(E, \mathbb{R})$ and

$$
\left\langle\Lambda^{\prime}(u), v\right\rangle=\int_{\Omega} \sum_{i=1}^{N} a_{i}\left(x, \frac{\partial u}{\partial x_{i}}\right) \frac{\partial v}{\partial x_{i}} d x
$$

for all $u, v \in E$.

Due to Lemma 9, a standard calculus leads to the facts that $I$ is well-defined on $E$ and $I \in \mathscr{C}^{1}(E, \mathbb{R})$ with the derivative given by

$$
\begin{aligned}
\left\langle I^{\prime}(u), v\right\rangle= & \int_{\Omega} \sum_{i=1}^{N} a_{i}\left(x, \frac{\partial u}{\partial x_{i}}\right) \frac{\partial v}{\partial x_{i}} d x \\
& +\int_{\Omega}|u|^{p_{M}(x)-2} u v d x-\int_{\Omega} f(x) v d x
\end{aligned}
$$

for all $u, v \in E$. Obviously, the weak solutions of (1) are the critical points of $I$; so by means of Theorem 7, we intend to prove the existence of critical points in order to deduce the existence of weak solutions.

Theorem 10. Assume (4)-(8) and $f \in L^{\infty}(\Omega)$. Then, there exists a unique weak solution of problem (1).

Let us start the proof by establishing some useful lemmas.

Lemma 11. If hypotheses (4)-(8) are fulfilled, then the functional $I$ is coercive.

Proof. Let $u \in E$ be such that $\|u\|_{E} \rightarrow \infty$. Using (7), we deduce that

$$
\Lambda(u) \geq \frac{1}{p_{M}^{+}} \sum_{i=1}^{N} \int_{\Omega}\left|\frac{\partial u}{\partial x_{i}}\right|^{p_{i}(x)} d x .
$$

We make the following notations:

$$
\begin{aligned}
& \mathscr{I}_{1}=\left\{i \in\{1, \ldots, N\}:\left|\frac{\partial u}{\partial x_{i}}\right|_{L^{p_{i}(\cdot)}(\Omega)} \leq 1\right\}, \\
& \mathscr{I}_{2}=\left\{i \in\{1, \ldots, N\}:\left|\frac{\partial u}{\partial x_{i}}\right|_{L^{p_{i}(\cdot)}(\Omega)}>1\right\} .
\end{aligned}
$$

We then have

$$
\begin{aligned}
\Lambda(u) \geq & \frac{1}{p_{M}^{+}} \sum_{i \in \mathscr{F}_{1}} \int_{\Omega}\left|\frac{\partial u}{\partial x_{i}}\right|^{p_{i}(x)} d x \\
& +\frac{1}{p_{M}^{+}} \sum_{i \in \mathscr{I}_{2}} \int_{\Omega}\left|\frac{\partial u}{\partial x_{i}}\right|^{p_{i}(x)} d x .
\end{aligned}
$$


Using (19), (20), and (21), we have

$$
\begin{aligned}
\Lambda(u) & \geq \frac{1}{p_{M}^{+}} \sum_{i \in \mathscr{F}_{1}}\left|\frac{\partial u}{\partial x_{i}}\right|_{p_{i}(\cdot)}^{p_{i}^{+}}+\frac{1}{p_{M}^{+}} \sum_{i \in \mathcal{F}_{2}}\left|\frac{\partial u}{\partial x_{i}}\right|_{p_{i}(\cdot)}^{p_{i}^{-}} \\
& \geq \frac{1}{p_{M}^{+}} \sum_{i \in F_{2}}\left|\frac{\partial u}{\partial x_{i}}\right|_{p_{i}(\cdot)}^{p_{i}^{-}} \\
& \geq \frac{1}{p_{M}^{+}} \sum_{i \in \mathscr{F}_{2}}\left|\frac{\partial u}{\partial x_{i}}\right|_{p_{i}(\cdot)}^{p_{m}^{-}} \\
& \geq \frac{1}{p_{M}^{+}}\left(\sum_{i=1}^{N}\left|\frac{\partial u}{\partial x_{i}}\right|_{p_{i}(\cdot)}^{p_{m}^{-}}-\sum_{i \in F_{1}}\left|\frac{\partial u}{\partial x_{i}}\right|_{p_{i}(\cdot)}^{p_{m}^{-}}\right) \\
& \geq \frac{1}{p_{M}^{+}}\left(\sum_{i=1}^{N}\left|\frac{\partial u}{\partial x_{i}}\right|_{p_{i}(\cdot)}^{p_{m}^{-}}-N\right) .
\end{aligned}
$$

By the generalized mean inequality or the Jensen's inequality applied to the convex function $z: \mathbb{R}^{+} \rightarrow \mathbb{R}^{+}, z(t)=$ $t^{p_{m}^{-}}, p_{m}^{-}>1$, we get

$$
\sum_{i=1}^{N}\left|\frac{\partial u}{\partial x_{i}}\right|_{p_{i}(\cdot)}^{p_{m}^{-}} \geq \frac{1}{N^{p_{m}^{-}-1}}\left(\sum_{i=1}^{N}\left|\frac{\partial u}{\partial x_{i}}\right|_{p_{i}(\cdot)}\right)^{p_{m}^{-}}
$$

thus,

$$
\Lambda(u) \geq \frac{1}{p_{M}^{+}}\left[\frac{1}{N^{p_{m}^{-}-1}}\left(\sum_{i=1}^{N}\left|\frac{\partial u}{\partial x_{i}}\right|_{p_{i}(\cdot)}\right)^{p_{m}^{-}}-N\right]
$$

Case $1\left(|u|_{p_{M}(.)} \geq 1\right)$. We have

$$
\begin{aligned}
I(u) \geq & \frac{1}{p_{M}^{+}}\left[\frac{1}{N^{p_{m}^{-}-1}}\left(\sum_{i=1}^{N}\left|\frac{\partial u}{\partial x_{i}}\right|_{p_{i}(\cdot)}\right)^{p_{m}^{-}}-N\right] \\
& +\frac{1}{p_{M}^{+}}|u|_{p_{M}(\cdot)}^{p_{M}^{-}}-\|f\|_{L^{\infty}(\Omega)}\|u\|_{L^{1}(\Omega)} \\
\geq & \frac{1}{p_{M}^{+}}\left[\frac{1}{N^{p_{m}^{-}-1}}\left(\sum_{i=1}^{N}\left|\frac{\partial u}{\partial x_{i}}\right|_{p_{i}(\cdot)}\right)^{p_{m}^{-}}+|u|_{p_{M}(\cdot)}^{p_{m}^{-}}\right] \\
\geq & \frac{1}{2 p_{m}^{-}-1} p_{M}^{+} \min \left(1, \frac{1}{N_{L^{p_{m}}-1}^{-}(\Omega)}\right) \\
& \cdot\left[\sum_{i=1}^{N}\left|\frac{\partial u}{\partial x_{i}}\right|_{p_{i}(\cdot)}+|u|_{p_{M}(\cdot)}\right]^{p_{M}^{+}} \\
& -C\|f\|_{L^{\infty}(\Omega)}\|u\|_{E}-\frac{N}{p_{M}^{+}} .
\end{aligned}
$$

Therefore,

$$
\begin{aligned}
I(u) \geq & \frac{1}{2^{p_{m}^{-}-1} p_{M}^{+}} \min \left(1, \frac{1}{N_{p_{m}^{-}-1}^{-}}\right)\|u\|_{E}^{p_{m}^{-}} \\
& -C\|f\|_{L^{\infty}(\Omega)}\|u\|_{E}-\frac{N}{p_{M}^{+}} .
\end{aligned}
$$

Case $2\left(|u|_{p_{M}(\cdot)}<1\right)$. Then $|u|_{p_{M}(\cdot)}^{p_{m}^{-}}-1<0$, and we get

$I(u)$

$$
\begin{aligned}
\geq & \frac{1}{p_{M}^{+}}\left[\frac{1}{N^{p_{m}^{-}-1}}\left(\sum_{i=1}^{N}\left|\frac{\partial u}{\partial x_{i}}\right|_{p_{i}(\cdot)}\right)^{p_{m}^{-}}+|u|_{p_{M}(\cdot)}^{p_{m}^{-}}-N-1\right] \\
& -\|f\|_{L^{\infty}(\Omega)}\|u\|_{L^{1}(\Omega)} \\
\geq & \frac{1}{p_{M}^{+}}\left[\frac{1}{N^{p_{m}^{-}-1}}\left(\sum_{i=1}^{N}\left|\frac{\partial u}{\partial x_{i}}\right|_{p_{i}(\cdot)}\right)^{p_{m}^{-}}+|u|_{p_{M}(\cdot)}^{p_{m}^{-}}\right] \\
& -C\|f\|_{L^{\infty}(\Omega)}\|u\|_{E}-\frac{N+1}{p_{M}^{+}} \\
\geq & \frac{1}{2 p_{m}^{-}-1} p_{M}^{+} \min \left(1, \frac{1}{N^{p_{m}^{-}-1}}\right)\left[\sum_{i=1}^{N}\left|\frac{\partial u}{\partial x_{i}}\right|_{p_{i}(\cdot)}+|u|_{p_{M}(\cdot)}\right]^{p_{m}^{-}} \\
& -C\|f\|_{L^{\infty}(\Omega)}\|u\|_{E}-\frac{N+1}{p_{M}^{+}} .
\end{aligned}
$$

So, we obtain

$$
\begin{aligned}
I(u) \geq & \frac{1}{2^{p_{m}^{-}-1} p_{M}^{+}} \min \left(1, \frac{1}{N p_{m}^{-}-1}\right)\|u\|_{E}^{p_{m}^{-}} \\
& -C\|f\|_{L^{\infty}(\Omega)}\|u\|_{E}-\frac{N+1}{p_{M}^{+}} .
\end{aligned}
$$

Then, letting $\|u\|_{E}$ goes to infinity in (66) and (68), we conclude that $I(u)$ reaches infinity. Thus, $I$ is coercive.

Lemma 12. The functional I is weakly lower semicontinuous.

Proof. By [33, Corollary III.8], it is enough to show that $I$ is lower semicontinuous. To this aim, fix $u \in E$ and $\epsilon>0$. Since for every $i \in\{1, \ldots, N\}, a_{i}(x, \cdot)$ is monotone, Theorem 6 yields

$$
\begin{gathered}
A_{i}\left(x, \frac{\partial v}{\partial x_{i}}\right)-A_{i}\left(x, \frac{\partial u}{\partial x_{i}}\right) \geq a_{i}\left(x, \frac{\partial u}{\partial x_{i}}\right)\left(\frac{\partial v}{\partial x_{i}}-\frac{\partial u}{\partial x_{i}}\right) \\
\Longrightarrow \sum_{i=1}^{N} \int_{\omega} A_{i}\left(x, \frac{\partial v}{\partial x_{i}}\right) d x \geq \sum_{i=1}^{N} \int_{\omega} A_{i}\left(x, \frac{\partial u}{\partial x_{i}}\right) d x \\
+\sum_{i=1}^{N} \int_{\omega} a_{i}\left(x, \frac{\partial u}{\partial x_{i}}\right)\left(\frac{\partial v}{\partial x_{i}}-\frac{\partial u}{\partial x_{i}}\right) d x
\end{gathered}
$$




$$
\begin{aligned}
\Longrightarrow & I(v) \geq I(u)+\sum_{i=1}^{N} \int_{\omega} a_{i}\left(x, \frac{\partial u}{\partial x_{i}}\right)\left(\frac{\partial v}{\partial x_{i}}-\frac{\partial u}{\partial x_{i}}\right) d x \\
& +\int_{\omega} \frac{1}{p_{M}(x)}\left(|v|^{p_{M}(x)}-|u|^{p_{M}(x)}\right) d x \\
& +\int_{\omega} f(x)(u-v) d x .
\end{aligned}
$$

Since the map $t \mapsto t^{p_{M}(x)}, t>0$ is convex, again by Theorem 6, we have

$$
|v|^{p_{M}(x)}-|u|^{p_{M}(x)} \geq p_{M}(x)|u|^{p_{M}(x)-2} u(v-u),
$$

then (69) becomes

$$
\begin{aligned}
I(v) \geq & I(u)+\sum_{i=1}^{N} \int_{\Omega} a_{i}\left(x, \frac{\partial u}{\partial x_{i}}\right)\left(\frac{\partial v}{\partial x_{i}}-\frac{\partial u}{\partial x_{i}}\right) d x \\
& +\int_{\Omega}|u|^{p_{M}(x)-2} u(v-u) d x \\
& +\int_{\Omega} f(x)(u-v) d x .
\end{aligned}
$$

Consider the second term in the right-hand side of (71). By (5) and Hölder type inequality, we have

$$
\begin{aligned}
& \sum_{i=1}^{N} \int_{\Omega} a_{i}\left(x, \frac{\partial u}{\partial x_{i}}\right)\left(\frac{\partial v}{\partial x_{i}}-\frac{\partial u}{\partial x_{i}}\right) d x \\
& \geq-\sum_{i=1}^{N} \int_{\Omega}\left|a_{i}\left(x, \frac{\partial u}{\partial x_{i}}\right)\right|\left|\frac{\partial v}{\partial x_{i}}-\frac{\partial u}{\partial x_{i}}\right| d x \\
& \geq-\max \left\{C_{1}, \ldots, C_{N}\right\} \\
& \cdot \sum_{i=1}^{N} \int_{\Omega}\left(j_{i}+\left|\frac{\partial u}{\partial x_{i}}\right|^{p_{i}(x)-1}\right)\left|\frac{\partial v}{\partial x_{i}}-\frac{\partial u}{\partial x_{i}}\right| d x \\
& \geq-K \sum_{i=1}^{N} \int_{\Omega} j_{i}(x)\left|\frac{\partial v}{\partial x_{i}}-\frac{\partial u}{\partial x_{i}}\right| d x \\
&-K \sum_{i=1}^{N} \int_{\Omega}\left|\frac{\partial u}{\partial x_{i}}\right|^{p_{i}(x)-1}\left|\frac{\partial v}{\partial x_{i}}-\frac{\partial u}{\partial x_{i}}\right|_{d x} \\
& \geq-K^{\prime} \sum_{i=1}^{N}\left|j_{i}\right|_{p_{i}^{\prime}(\cdot)}\left|\frac{\partial v}{\partial x_{i}}-\frac{\partial u}{\partial x_{i}}\right|_{p_{i}(\cdot)} \\
&-\left.K^{\prime} \sum_{i=1}^{N}|| \frac{\partial u}{\partial x_{i}}\right|^{p_{i}(x)-1}||_{p_{i}^{\prime}(\cdot)}\left|\frac{\partial v}{\partial x_{i}}-\frac{\partial u}{\partial x_{i}}\right|_{p_{i}(\cdot)} \\
& \geq-K^{\prime} \max _{i}\left\{\left.\left|j_{i}\right|_{p_{i}^{\prime}(\cdot)}|| \frac{\partial u}{\partial x_{i}}\right|^{p_{i}(x)-1} \mid\right. \\
& \cdot \sum_{i=1}^{N}\left|\frac{\partial v}{\partial x_{i}}-\frac{\partial u}{\partial x_{i}}\right|_{p_{i}(\cdot)} \geq-C_{1} \sum_{i=1}^{N}\left|\frac{\partial u}{\partial x_{i}}-\frac{\partial v}{\partial x_{i}}\right|_{p_{i}(\cdot)}
\end{aligned}
$$

For the fourth term in the right-hand side of (71), we have

$$
\begin{aligned}
\int_{\Omega} f(x)(u-v) d x & \geq-\int_{\Omega}|f(x)||u-v| d x \\
& \geq\|f\|_{L^{\infty}(\Omega)}\|u-v\|_{L^{1}(\Omega)} \\
& \geq-C_{2}\|u-v\|_{E} .
\end{aligned}
$$

The third term in the right-hand side of (71) gives by using Hölder type inequality

$$
\begin{aligned}
& \int_{\Omega}|u|^{p_{M}(x)-2} u(v-u) d x \\
& \quad \geq-\int_{\Omega}|u|^{p_{M}(x)-1}|u-v| d x \\
& \geq-\left.\left.C^{\prime}|| u\right|^{p_{M}(x)-1}\right|_{P_{M}^{\prime}(\cdot)}|u-v|_{p_{M}(\cdot)} \\
& \geq-C_{3}|u-v|_{p_{M}(\cdot)} .
\end{aligned}
$$

Gathering these inequalities, it follows that

$$
I(v) \geq I(u)-C\|u-v\|_{E} \geq I(u)-\epsilon,
$$

for every $v \in E$ such that $\|u-v\|_{E}<\epsilon / C$. Thus, $I$ is lower semicontinuous.

\section{Proof of Theorem 10. Consider the following}

Step 1. Existence of weak solutions. The proof follows directly from Lemmas 11 and 12 and Theorem 7.

Step 2. Uniqueness of weak solution. Let $u, v \in E$ be two weak solutions of problem (1). Choosing a test function in (54), $\varphi=$ $v-u$ for the weak solution $u$ and $\varphi=u-v$ for the weak solution $v$, we get

$$
\begin{array}{r}
\int_{\Omega} \sum_{i=1}^{N} a_{i}\left(x, \frac{\partial u}{\partial x_{i}}\right) \frac{\partial(v-u)}{\partial x_{i}} d x \\
+\int_{\Omega}|u|^{p_{M}(x)-2} u(v-u) d x \\
-\int_{\Omega} f(x)(v-u) d x=0, \\
\int_{\Omega} \sum_{i=1}^{N} a_{i}\left(x, \frac{\partial v}{\partial x_{i}}\right) \frac{\partial(u-v)}{\partial x_{i}} d x \\
+\int_{\Omega}|v|^{p_{M}(x)-2} v(u-v) d x \\
-\int_{\Omega} f(x)(u-v) d x=0 .
\end{array}
$$

Summing up (76) and (77), we obtain

$$
\begin{aligned}
& \int_{\Omega} \sum_{i=1}^{N}\left(a_{i}\left(x, \frac{\partial u}{\partial x_{i}}\right)-a_{i}\left(x, \frac{\partial v}{\partial x_{i}}\right)\right) \frac{\partial(u-v)}{\partial x_{i}} d x \\
& \quad+\int_{\Omega} \frac{1}{p_{M}(x)}\left(|u|^{p_{M}(x)-2} u-|v|^{p_{M}(x)-2} v\right)(u-v) d x=0 .
\end{aligned}
$$


Thus, by the monotonicity of the functions $a_{i}(x, \cdot)$ and $t \mapsto$ $|t|^{p_{M}(x)-2} t$, we deduce that $u=v$ almost everywhere.

3.2. Entropy Solutions. First of all, we define a space in which we will look for entropy solutions. We define the space $\mathscr{T}^{1, \vec{p}(\cdot)}(\Omega)$ as the set of every measurable function $u: \Omega \rightarrow \mathbb{R}$ which satisfies for every $k>0, T_{k}(u) \in W^{1, \vec{p}(\cdot)}(\Omega)$.

Lemma 13 (see $[34,35])$. Let $u \in \mathscr{T}^{1, \vec{p}(\cdot)}(\Omega)$. Then, there exists a unique measurable function $v_{i}: \Omega \rightarrow \mathbb{R}$ such that

$$
\begin{aligned}
& v_{i} \chi_{\{|u|<k\}} \\
& =\frac{\partial T_{k}(u)}{\partial x_{i}} \quad \text { for a.e. } x \in \Omega, \forall k>0, i \in\{1, \ldots, N\},
\end{aligned}
$$

where $\chi_{A}$ denotes the characteristic function of a measurable set $A$. The functions $v_{i}$ are called the weak partial gradients of $u$ and are still denoted $\partial u / \partial x_{i}$. Moreover, if $u$ belongs to $W^{1, \vec{p}(\cdot)}(\Omega)$, then $v_{i} \in L^{p_{i}(\cdot)}(\Omega)$ and coincides with the standard distributional gradient of $u$, that is, $v_{i}=\partial u / \partial x_{i}$.

Definition 14. We define the space $\mathscr{T}_{\mathscr{H}}^{1, \vec{p}(\cdot)}(\Omega)$ as the set of function $u \in \mathscr{T}^{1, \vec{p}(\cdot)}(\Omega)$ such that there exists a sequence $\left(u_{n}\right)_{n} \subset W^{1, \vec{p}(\cdot)}(\Omega)$ satisfying

(a) $u_{n} \rightarrow u$ a.e. in $\Omega$,

(b) $\partial T_{k}\left(u_{n}\right) / \partial x_{i} \rightarrow \partial T_{k}(u) / \partial x_{i}$ in $L^{1}(\Omega)$, for all $k>0$.

Definition 15. A measurable function $u$ is an entropy solution of (1) if $u \in \mathscr{T}_{\mathscr{H}}^{1, \vec{p}(\cdot)}(\Omega)$ and for every $k>0$,

$$
\begin{gathered}
\sum_{i=1}^{N} \int_{\Omega} a_{i}\left(x, \frac{\partial u}{\partial x_{i}}\right) \frac{\partial}{\partial x_{i}} T_{k}(u-\varphi) d x \\
\quad+\int_{\Omega}|u|^{p_{M}(x)-2} u T_{k}(u-\varphi) d x \\
\leq \int_{\Omega} f(x) T_{k}(u-\varphi) d x
\end{gathered}
$$

for all $\varphi \in W^{1, \vec{p}^{(\cdot)}}(\Omega) \cap L^{\infty}(\Omega)$.

Our main result in this section is the following.

Theorem 16. Assume (4)-(8) and $f \in L^{1}(\Omega)$. Then, there exists a unique entropy solution $u$ to problem (1).

Proof. The proof of this Theorem will be done in three steps.

Step 1 (a priori estimates).

Lemma 17. Assume (4)-(8) and $f \in L^{1}(\Omega)$. Let $u$ be an entropy solution of (1). If there exists a positive constant $M$ such that

$$
\sum_{i=1}^{N} \int_{\{|u|>t\}} t^{q_{i}(x)} d x \leq M, \quad \forall t>0,
$$

then

$$
\sum_{i=1}^{N} \int_{\left\{\left|\left(\partial / \partial x_{i}\right) u\right|^{\alpha_{i}(\cdot)}>t\right\}} t^{q_{i}(x)} d x \leq\|f\|_{1}+M \quad \forall t>0,
$$

where $\alpha_{i}(\cdot)=p_{i}(\cdot) /\left(q_{i}(\cdot)+1\right)$, for all $i=1, \ldots, N$.

Proof. Take $\varphi=0$ in (80), we have

$$
\begin{aligned}
\sum_{i=1}^{N} \int_{\Omega} a_{i} & \left(x, \frac{\partial}{\partial x_{i}} T_{t}(u)\right) \cdot \frac{\partial}{\partial x_{i}} T_{t}(u) d x \\
& +\int_{\Omega}|u|^{p_{M}(x)-2} u T_{t}(u) d x \leq \int_{\Omega} f(x) T_{t}(u) d x .
\end{aligned}
$$

Since the second term in the previous inequality is nonnegative, it follows that

$$
\begin{gathered}
\sum_{i=1}^{N} \int_{\Omega} a_{i}\left(x, \frac{\partial}{\partial x_{i}} T_{t}(u)\right) \cdot \frac{\partial}{\partial x_{i}} T_{t}(u) d x \\
\leq \int_{\Omega} f(x) T_{t}(u) d x
\end{gathered}
$$

According to (7), we deduce that

$$
\sum_{i=1}^{N} \int_{\Omega}\left|\frac{\partial}{\partial x_{i}} T_{t}(u)\right|^{p_{i}(x)} d x \leq t\|f\|_{1}, \quad \forall t>0 .
$$

Therefore, defining $\psi:=T_{t}(u) / t$, we have for all $t>0$,

$$
\begin{aligned}
\sum_{i=1}^{N} \int_{\Omega} t^{p_{i}(x)-1}\left|\frac{\partial}{\partial x_{i}} \psi\right|^{p_{i}(x)} d x & \\
= & \sum_{i=1}^{N} \frac{1}{t} \int_{\Omega}\left|\frac{\partial}{\partial x_{i}} T_{t}(u)\right|^{p_{i}(x)} d x \leq\|f\|_{1} .
\end{aligned}
$$

From the previous inequality, the definition of $\alpha_{i}(\cdot)$ and (81), we have

$$
\begin{aligned}
& \sum_{i=1}^{N} \int_{\left\{\left|\left(\partial / \partial x_{i}\right) u\right|^{\alpha_{i}(\cdot)}>t\right\}} t^{q_{i}(x)} d x \\
& \leq \sum_{i=1}^{N} \int_{\left\{\left|\left(\partial / \partial x_{i}\right) u\right|^{\alpha_{i}(\cdot)}>t\right\} \cap\{|u| \leq t\}} t^{q_{i}(x)} d x \\
& \quad+\sum_{i=1}^{N} \int_{\{|u|>t\}} t^{q_{i}(x)} d x
\end{aligned}
$$$$
\leq \sum_{i=1}^{N} \int_{\{|u| \leq t\}} t^{q_{i}(x)}\left(\frac{\left|\left(\partial / \partial x_{i}\right) u\right|^{\alpha_{i}(x)}}{t}\right)^{p_{i}(x) / \alpha_{i}(x)} d x+M
$$$$
\leq \sum_{i=1}^{N} \int_{\left\{\left|\left(\partial / \partial x_{i}\right) u\right|^{\alpha_{i}(\cdot)}>t ;|u| \leq t\right\}} t^{q_{i}(x)-\left(p_{i}(x) / \alpha_{i}(x)\right)}\left|\frac{\partial u}{\partial x_{i}}\right|^{p_{i}(x)} d x+M
$$$$
\leq \sum_{i=1}^{N} \frac{1}{t} \int_{\left\{\left|\left(\partial / \partial x_{i}\right) u\right|^{\alpha_{i}(\cdot)}>t ;|u| \leq t\right\}}\left|\frac{\partial u}{\partial x_{i}}\right|^{p_{i}(x)} d x
$$$$
+M \leq\|f\|_{1}+M \text {. }
$$ 
Lemma 18. Assume (4)-(8) and $f \in L^{1}(\Omega)$. Let u be an entropy solution of (1), then

$$
\frac{1}{h} \sum_{i=1}^{N} \int_{\{|u| \leq h\}}\left|\frac{\partial}{\partial x_{i}} T_{h}(u)\right|^{p_{i}(x)} d x \leq M
$$

for every $h>0$, with $M$ a positive constant. Moreover, we have

$$
\left\||u|^{p_{M}(x)-2} u\right\|_{1}=\left\||u|^{p_{M}(x)-1}\right\|_{1} \leq\|f\|_{1}
$$

and there exists a constant $D>0$ which depends on $f$ and $\Omega$ such that

$$
\text { meas }\{|u|>h\} \leq \frac{D}{h^{P^{-1}}}, \quad \forall h>0 .
$$

Proof. Taking $\varphi=0$ in the entropy inequality (80) and using (7), we obtain

$$
\begin{gathered}
\sum_{i=1}^{N} \int_{\{|u| \leq h\}}\left|\frac{\partial}{\partial x_{i}} T_{h}(u)\right|^{p_{i}(x)} d x \leq h\|f\|_{1} \leq M h, \\
\int_{\Omega}|u|^{p_{M}(x)-2} u T_{h}(u) d x \leq h\|f\|_{1},
\end{gathered}
$$

for all $h>0$. This yields

$$
\int_{\{|u|>h\}}|u|^{p_{M}(x)-2} u T_{h}(u) d x \leq h\|f\|_{1} .
$$

As $u T_{h}(u) \chi_{\{|u|>h\}}=h|u| \chi_{\{|u|>h\}}$, we get from the previous inequality by using Fatou's lemma

$$
\int_{\Omega}|u|^{p_{M}(x)-2}|u| d x \leq\|f\|_{1} .
$$

Now, since $\left|T_{h}(u)\right| \leq|u|$ we have

$$
\int_{\Omega}\left|T_{h}(u)\right|^{p_{M}(x)-1} d x \leq \int_{\Omega}|u|^{p_{M}(x)-1} d x \leq\|f\|_{1} .
$$

We deduce that

$$
\int_{\Omega}\left|T_{h}(u)\right|^{p_{M}^{-}-1} d x \leq D(f, \Omega) .
$$

Indeed,

$$
\begin{aligned}
\int_{\Omega}\left|T_{h}(u)\right|^{p_{M}^{-}-1} d x & \\
\leq & \int_{\left\{\left|T_{h}(u)\right| \leq 1\right\}}\left|T_{h}(u)\right|^{p_{M}^{-}-1} d x \\
& +\int_{\left\{\left|T_{h}(u)\right|>1\right\}}\left|T_{h}(u)\right|^{p_{M}^{-}-1} d x \\
\leq & \operatorname{meas}(\Omega)+\int_{\Omega}\left|T_{h}(u)\right|^{p_{M}(x)-1} d x \\
\leq & \operatorname{meas}(\Omega)+\|f\|_{1} .
\end{aligned}
$$

From aforementioned, we get

$$
\int_{\{|u|>h\}}\left|T_{h}(u)\right|^{p_{M}^{-}-1} d x \leq D(f, \Omega) .
$$

Therefore,

$$
h^{p_{M}^{-1}} \text { meas }\{|u|>h\} \leq D(f, \Omega)
$$

which implies

$$
\text { meas }\{|u|>h\} \leq \frac{D(f, \Omega)}{h^{p^{-1}}} .
$$

Lemma 19. If $u$ is an entropy solution of (1) then there exists a constant $C>0$ such that

$$
\int_{\Omega}\left|T_{k}(u)\right|^{P_{M}^{-}} d x+\sum_{i=1}^{N} \int_{\{|u| \leq k\}}\left|\frac{\partial u}{\partial x_{i}}\right|^{P_{i}^{-}} d x \leq C(k+1),
$$

$\forall k>0$.

Proof. Taking $\varphi=0$ in the entropy inequality (80) and using (7), we get

$$
\begin{aligned}
& \int_{\Omega}|u|^{p_{M}(x)-2} u T_{k}(u) d x \\
& \quad+\sum_{i=1}^{N} \int_{\{|u| \leq k\}}\left|\frac{\partial}{\partial x_{i}} T_{k}(u)\right|^{p_{i}(x)} d x \leq k\|f\|_{1} .
\end{aligned}
$$

Note that

$$
\begin{aligned}
& \sum_{i=1}^{N} \int_{\{|u| \leq k\}}\left|\frac{\partial}{\partial x_{i}} T_{k}(u)\right|^{p_{i}^{-}} d x \\
& =\sum_{i=1}^{N} \int_{\left\{|u| \leq k,\left|\partial u / \partial x_{i}\right| \leq 1\right\}}\left|\frac{\partial}{\partial x_{i}} T_{k}(u)\right|^{p_{i}^{-}} d x \\
& \quad+\sum_{i=1}^{N} \int_{\left\{|u| \leq k,\left|\partial u / \partial x_{i}\right|>1\right\}}\left|\frac{\partial}{\partial x_{i}} T_{k}(u)\right|^{p_{i}^{-}} d x \\
& \leq N \operatorname{meas}(\Omega)+\sum_{i=1}^{N} \int_{\left\{|u| \leq k,\left|\partial u / \partial x_{i}\right|>1\right\}}\left|\frac{\partial}{\partial x_{i}} T_{k}(u)\right|^{p_{i}(x)} d x \\
& \quad \leq N \operatorname{meas}(\Omega)+\sum_{i=1}^{N} \int_{\{|u| \leq k\}}\left|\frac{\partial}{\partial x_{i}} T_{k}(u)\right|^{p_{i}(x)} d x, \\
& \int_{\Omega}\left|T_{k}(u)\right|^{p_{M}^{-}} d x \\
& \quad \leq \int_{\left\{\left|T_{k}(u)\right| \leq 1\right\}}\left|T_{k}(u)\right|^{p_{M}^{-}} d x+\int_{\left\{\left|T_{k}(u)\right|>1\right\}}\left|T_{k}(u)\right|^{p_{M}^{-}} d x \\
& \quad \leq \operatorname{meas}(\Omega)+\int_{\Omega}\left|T_{k}(u)\right|^{p_{M}(x)} d x \\
& \quad \leq \operatorname{meas}(\Omega)+\int_{\Omega}|u|^{p_{M}(x)-2} u T_{k}(u) d x .
\end{aligned}
$$


Therefore, we deduce according to (101) that

$$
\begin{aligned}
& \int_{\Omega}\left|T_{k}(u)\right|^{P_{M}^{-}} d x+\sum_{i=1}^{N} \int_{\{|u| \leq k\}}\left|\frac{\partial u}{\partial x_{i}}\right|^{p_{i}^{-}} d x \\
& \leq(N+1) \operatorname{meas}(\Omega)+k\|f\|_{1}, \quad \forall k>0 .
\end{aligned}
$$

Lemma 20. If $u$ is an entropy solution of (1) then

$$
\rho_{p^{\prime}(\cdot)}\left(\left|\frac{\partial}{\partial x_{i}} u\right|^{p_{i}(x)-1} \chi_{F}\right) \leq C, \quad \forall i=1, \ldots, N,
$$

where $F=\{h<|u| \leq h+t\}, h>0, t>0$.

Proof. Taking $\varphi=T_{h}(u)$ as a test function in the entropy inequality (80), we get

$$
\begin{gathered}
\sum_{i=1}^{N} \int_{\Omega} a_{i}\left(x, \frac{\partial}{\partial x_{i}} u\right) \cdot \frac{\partial}{\partial x_{i}} T_{t}\left(u-T_{h}(u)\right) d x \\
\quad+\int_{\Omega}|u|^{p_{M}(x)-2} u T_{t}\left(u-T_{h}(u)\right) d x \\
\leq \int_{\Omega} f(x) T_{t}\left(u-T_{h}(u)\right) d x .
\end{gathered}
$$

It follows by using (7) that

$$
\int_{F}\left|\frac{\partial}{\partial x_{i}} u\right|^{p_{i}(x)} d x \leq t\|f\|_{1}
$$

Therefore,

$$
\rho_{p^{\prime}(\cdot)}\left(\left|\frac{\partial}{\partial x_{i}} u\right|^{p_{i}(x)-1} \chi_{F}\right) \leq C, \quad \forall i=1, \ldots, N .
$$

Lemma 21. If $u$ is an entropy solution of (1) then

$$
\lim _{h \rightarrow+\infty} \int_{\Omega}|f| \chi_{\{|u|>h-t\}} d x=0
$$

where $h>0, t>0$.

Proof. By Lemma 18, we deduce that

$$
\lim _{h \rightarrow+\infty}|f| \chi_{\{|u|>h-t\}}=0
$$

and as $f \in L^{1}(\Omega)$, it follows by using the Lebesgue dominated convergence theorem that

$$
\lim _{h \rightarrow+\infty} \int_{\Omega}|f| \chi_{\{|u|>h-t\}} d x=0 .
$$

The proof of the following lemma can be found in [1].
Lemma 22. Assume (4)-(8) and $f \in L^{1}(\Omega)$. Let $u$ be an entropy solution of (1), then

$$
\text { meas }\left\{\left|\frac{\partial}{\partial x_{i}} u\right|>h\right\} \leq \frac{D^{\prime}}{h^{1 /\left(P_{M}^{-}\right)^{\prime}}}, \quad \forall h \geq 1, \forall i=1, \ldots, N
$$

where $D^{\prime}$ is a positive constant which depends on $f$ and $p_{M}^{-}$.

Step 2 (uniqueness of entropy solution). The proof of the uniqueness of entropy solutions follows the same techniques by Ouaro [20] (see also [35]). Indeed, let $h>0$ and $u, v$ be two entropy solutions of (1). We write the entropy inequality (54) corresponding to the solution $u$, with $T_{h}(v)$ as test function, and to the solution $v$, with $T_{h}(u)$ as test function. Upon addition, we get

$$
\begin{aligned}
& \int_{\left\{\left|u-T_{h}(v)\right| \leq t\right\}} \sum_{i=1}^{N} a_{i}\left(x, \frac{\partial}{\partial x_{i}} u\right) \\
& \quad \cdot \frac{\partial}{\partial x_{i}}\left(u-T_{h}(v)\right) d x \\
& \quad+\int_{\left\{\left|v-T_{h}(u)\right| \leq t\right\}} \sum_{i=1}^{N} a_{i}\left(x, \frac{\partial}{\partial x_{i}} v\right) \\
& \quad \cdot \frac{\partial}{\partial x_{i}}\left(v-T_{h}(u)\right) d x \\
& \quad+\int_{\Omega}|u|^{p_{M}(x)-2} u T_{t}\left(u-T_{h}(v)\right) d x \\
& \quad+\int_{\Omega}|v|^{p_{M}(x)-2} v T_{t}\left(v-T_{h}(u)\right) d x \\
& \leq \int_{\Omega} f(x)\left(T_{t}\left(u-T_{h}(v)\right)+T_{t}\left(v-T_{h}(u)\right)\right) d x
\end{aligned}
$$

Define

$$
\begin{gathered}
E_{1}:=\{|u-v| \leq t,|v| \leq h\}, \\
E_{2}:=E_{1} \cap\{|u| \leq h\}, \\
E_{3}:=E_{1} \cap\{|u|>h\} .
\end{gathered}
$$

We start with the first integral in (112). By (7), we have

$$
\begin{gathered}
\int_{\left\{\left|u-T_{h} v\right| \leq t\right\}} \sum_{i=1}^{N} a_{i}\left(x, \frac{\partial}{\partial x_{i}} u\right) \cdot \frac{\partial}{\partial x_{i}}\left(u-T_{h}(v)\right) d x \\
\geq \int_{E_{2}} \sum_{i=1}^{N} a_{i}\left(x, \frac{\partial}{\partial x_{i}} u\right) \cdot \frac{\partial}{\partial x_{i}}(u-v) d x \\
-\int_{E_{3}} \sum_{i=1}^{N} a_{i}\left(x, \frac{\partial}{\partial x_{i}} u\right) \cdot \frac{\partial}{\partial x_{i}} v d x .
\end{gathered}
$$


Using (5) and Proposition 1, we estimate the last integral in (114) as follows:

$$
\begin{aligned}
\left|\int_{E_{3}} \sum_{i=1}^{N} a_{i}\left(x, \frac{\partial}{\partial x_{i}} u\right) \cdot \frac{\partial}{\partial x_{i}} v d x\right| \\
\leq C_{1} \int_{E_{3}} \sum_{i=1}^{N}\left(j_{i}(x)+\left|\frac{\partial}{\partial x_{i}} u\right|^{p_{i}(x)-1}\right)\left|\frac{\partial}{\partial x_{i}} v\right| d x \\
\leq C \sum_{i=1}^{N}\left(|j|_{p_{i}^{\prime}(\cdot)}+\left.\left.|| \frac{\partial}{\partial x_{i}} u\right|^{p_{i}(x)-1}\right|_{p_{i}^{\prime}(\cdot),\{h<|u| \leq h+t\}}\right) \\
\quad \cdot\left|\frac{\partial}{\partial x_{i}} v\right|_{p_{i}(\cdot),\{h-t<|v| \leq h\}},
\end{aligned}
$$

where

$$
\begin{aligned}
\left.\left.|| \frac{\partial}{\partial x_{i}} u\right|^{p_{i}(x)-1}\right|_{p_{i}^{\prime}(\cdot),\{h<|u| \leq h+t\}} \\
=\left\|\left.\frac{\partial}{\partial x_{i}} u\right|^{p_{i}(x)-1}\right\|_{L^{p_{i}^{\prime} \cdot(\{h<|u| \leq h+t\})}} .
\end{aligned}
$$

For all $i=1, \ldots, N$, the quantity $\left(\left|j_{i}\right|_{p_{i}^{\prime}(\cdot)}+\right.$ $\left.\|\left.\left.\left(\partial / \partial x_{i}\right) u\right|^{p_{i}(x)-1}\right|_{p_{i}^{\prime}(\cdot),\{h<|u| \leq h+t\}}\right)$ is finite according to relations (18), (19) and Lemma 20. The quantity $\left|\left(\partial / \partial x_{i}\right) v\right|_{p_{i}(\cdot),\{h-t<|v| \leq h\}}$ converges to zero as $h$ goes to infinity according to Lemma 21. Then, the last expression in (115) converges to zero as $h$ tends to infinity. Therefore, from (114), we obtain

$$
\begin{array}{r}
\int_{\left\{\left|u-T_{h} v\right| \leq t\right\}} \sum_{i=1}^{N} a_{i}\left(x, \frac{\partial}{\partial x_{i}} u\right) \cdot \frac{\partial}{\partial x_{i}}\left(u-T_{h}(v)\right) d x \\
\geq I_{h}+\int_{E_{2}} \sum_{i=1}^{N} a_{i}\left(x, \frac{\partial}{\partial x_{i}} u\right) \cdot \frac{\partial}{\partial x_{i}}(u-v) d x,
\end{array}
$$

where $I_{h}$ converges to zero as $h$ tends to infinity. We may adopt the same procedure to treat the second term in (112) to obtain

$$
\begin{array}{r}
\int_{\left\{\left|v-T_{h}(u)\right| \leq t\right\}} \sum_{i=1}^{N} a_{i}\left(x, \frac{\partial}{\partial x_{i}} v\right) \cdot \frac{\partial}{\partial x_{i}}\left(v-T_{h}(u)\right) d x \\
\geq J_{h}-\int_{E_{2}} \sum_{i=1}^{N} a_{i}\left(x, \frac{\partial}{\partial x_{i}} v\right) \cdot \frac{\partial}{\partial x_{i}}(u-v) d x
\end{array}
$$

where $J_{h}$ converges to zero as $h$ tends to infinity.

For the two other terms in the left-hand side of (112), we denote

$$
\begin{aligned}
K_{h}= & \int_{\Omega}|u|^{p_{M}(x)-2} u T_{t}\left(u-T_{h}(v)\right) d x \\
& +\int_{\Omega}|v|^{p_{M}(x)-2} v T_{t}\left(v-T_{h}(u)\right) d x .
\end{aligned}
$$

We have $|u|^{p_{M}(x)-2} u T_{t}\left(u-T_{h}(v)\right) \rightarrow|u|^{p_{M}(x)-2} u T_{t}(u-v)$ a.e. as $h$ goes to infinity and

$$
\left.|u|^{p_{M}(x)-2} u T_{t}\left(u-T_{h}(v)\right)|\leq t| u\right|^{p_{M}(x)-2} u \in L^{1}(\Omega) .
$$

Then, by the Lebesgue dominated convergence theorem, we obtain

$$
\begin{aligned}
\int_{\Omega}|u|^{p_{M}(x)-2} u T_{t}\left(u-T_{h}(v)\right) d x & \\
& \longrightarrow \int_{\Omega}|u|^{p_{M}(x)-2} u T_{t}(u-v) d x, \quad \text { as } h \longrightarrow \infty .
\end{aligned}
$$

In the same way, we get

$$
\begin{aligned}
\int_{\Omega}|v|^{p_{M}(x)-2} v T_{t}\left(v-T_{h}(u)\right) d x & \\
& \longrightarrow \int_{\Omega}|v|^{p_{M}(x)-2} v T_{t}(v-u) d x, \quad \text { as } h \longrightarrow \infty .
\end{aligned}
$$

Therefore,

$$
\lim _{h \rightarrow \infty} K_{h}=\int_{\Omega}\left(|u|^{p_{M}(x)-2} u-|v|^{p_{M}(x)-2} v\right) T_{t}(u-v) d x
$$

Furthermore, consider the right-hand side of inequality (112). We have

$$
\lim _{h \rightarrow \infty} \int_{\Omega} f(x)\left(T_{t}\left(u-T_{h}(v)\right)+T_{t}\left(v-T_{h}(u)\right)\right) d x=0 .
$$

Indeed,

$$
\begin{gathered}
f(x)\left(T_{t}\left(u-T_{h}(v)\right)+T_{t}\left(v-T_{h}(u)\right)\right) \\
\longrightarrow f(x)\left(T_{t}(u-v)+T_{t}(v-u)\right)=0 \\
\text { a.e. in } \Omega \text { as } h \longrightarrow \infty, \\
\left|f(x)\left(T_{t}\left(u-T_{h}(v)\right)+T_{t}\left(v-T_{h}(u)\right)\right)\right| \\
\leq 2 t|f(x)| \in L^{1}(\Omega),
\end{gathered}
$$

so that we are able to apply the Lebesgue dominated convergence theorem. Then, we deduce from relations (112)-(124) after passing to the limit as $h \rightarrow \infty$ in (112) the following:

$$
\begin{gathered}
\sum_{i=1}^{N} \int_{\{|u-v| \leq t\}}\left(a_{i}\left(x, \frac{\partial}{\partial x_{i}} u\right)-a_{i}\left(x, \frac{\partial}{\partial x_{i}} v\right)\right) \cdot \frac{\partial}{\partial x_{i}}(u-v) \\
+\int_{\Omega}\left(|u|^{p_{M}(x)-2} u-|v|^{p_{M}(x)-2} v\right) T_{t}(u-v) \leq 0 .
\end{gathered}
$$

Using (6) and as $t \mapsto|t|^{p_{M}(x)-2} t$ is monotone, we deduce from (126) that

$$
\int_{\Omega}\left(|u|^{p_{M}(x)-2} u-|v|^{p_{M}(x)-2} v\right) T_{t}(u-v) d x \leq 0 .
$$

Since $p_{M}^{-}>1$, the following relation is true for any $\xi, \eta \in \mathbb{R}$, $\xi \neq \eta$ (cf. [12])

$$
\left(|\xi|^{p_{M}(x)-2} \xi-|\eta|^{p_{M}(x)-2} \eta\right)(\xi-\eta)>0 .
$$


Therefore, from (127), we get that $\left(|u|^{p_{M}(x)-2} u-|v|^{p_{M}(x)-2} v\right)$ $T_{t}(u-v)=0$ a.e. in $\Omega$, which means that for all $t \in \mathbb{R}^{+}$, there exists $\Omega_{t} \subset \Omega$ with meas $\left(\Omega_{t}\right)=0$ such that for all $x \in \Omega \backslash \Omega_{t}$,

$$
\left(|u|^{p_{M}(x)-2} u-|v|^{p_{M}(x)-2} v\right) T_{t}(u-v)=0 .
$$

Therefore,

$$
\begin{array}{r}
\left(|u|^{p_{M}(x)-2} u-|v|^{p_{M}(x)-2} v\right)(u-v)=0, \\
\forall x \in \Omega \backslash \bigcup_{t \in \mathbb{N}^{*}} \Omega_{t} .
\end{array}
$$

Now, using (128) and (130), we obtain

$$
u_{1}=u_{2} \quad \text { a.e. in } \Omega \text {. }
$$

Step 3 (Existence of entropy solutions). Let $\left(f_{n}\right)_{n \in \mathbb{N}^{*}}$ be a sequence of bounded functions, strongly converging to $f \in$ $L^{1}(\Omega)$ and such that

$$
\left\|f_{n}\right\|_{1} \leq\|f\|_{1}, \quad \forall n \in \mathbb{N}^{*} .
$$

We consider the problem

$$
\begin{gathered}
-\sum_{i=1}^{N} \frac{\partial}{\partial x_{i}} a_{i}\left(x, \frac{\partial}{\partial x_{i}} u_{n}\right)+\left|u_{n}\right|^{p_{M}(x)-2} u_{n}=f_{n} \quad \text { in } \Omega, \\
\sum_{i=1}^{N} a_{i}\left(x, \frac{\partial}{\partial x_{i}} u_{n}\right) v_{i}=0 \quad \text { on } \partial \Omega .
\end{gathered}
$$

It follows from Theorem 10 that problem (133) admits a unique weak solution $u_{n} \in W^{1, \vec{p}(\cdot)}(\Omega)$ which satisfies

$$
\begin{gathered}
\sum_{i=1}^{N} \int_{\Omega} a_{i}\left(x, \frac{\partial u_{n}}{\partial x_{i}}\right) \frac{\partial}{\partial x_{i}} \varphi d x+\int_{\Omega}\left|u_{n}\right|^{p_{M}(x)-2} u_{n} \varphi d x \\
=\int_{\Omega} f_{n}(x) \varphi d x,
\end{gathered}
$$

for all $\varphi \in W^{1, \vec{p}(\cdot)}(\Omega)$.

Our interest is to prove that these approximated solutions $u_{n}$ tend, as $n$ goes to infinity, to a measurable function $u$ which is an entropy solution of the problem (1). We announce the following important lemma, useful to get some convergence results.

Lemma 23. If $u_{n}$ is a weak solution of (126) then there exist some constants $C_{1}, C_{2}>0$ such that

(i) $\left\|u_{n}\right\|_{\mathscr{M}^{\tilde{p}}(\Omega)} \leq C_{1}$,

(ii) $\left\|\partial u_{n} / \partial x_{i}\right\|_{\mathscr{M}^{p_{i}^{-} q / \bar{p}}(\Omega)} \leq C_{2}$, for all $i=1, \ldots, N$.

Proof. (i) is a consequence of Lemmas 19 and 5 by using $T_{k}\left(u_{n}\right)$ for all $k>0$ as a test function in (134).

(ii) We first use $T_{\gamma}\left(u_{n}\right)$ for all $\gamma>0$ as a test function in (134) to get

$$
\sum_{i=1}^{N} \int_{\{|u| \leq \gamma\}}\left|\frac{\partial u}{\partial x_{i}}\right|^{p_{i}^{-}} d x \leq C(\gamma+1) .
$$

Then, let $\lambda_{\left|\partial u_{n} / \partial x_{i}\right|}(\alpha)=\operatorname{meas}\left\{x \in \Omega:\left|\partial u_{n} / \partial x_{i}\right|>\alpha\right\}$ for all $i=1, \ldots, N$, we have for any $\alpha>1, \gamma>0$,

$$
\begin{aligned}
\lambda_{\left|\partial u_{n} / \partial x_{i}\right|}(\alpha) \leq & \text { meas }\left\{x \in \Omega:\left|\frac{\partial u_{n}}{\partial x_{i}}\right|>\alpha,\left|u_{n}\right| \leq \gamma\right\} \\
& + \text { meas }\left\{x \in \Omega:\left|\frac{\partial u_{n}}{\partial x_{i}}\right|>\alpha,\left|u_{n}\right|>\gamma\right\} \\
\leq & \int_{\left\{\left|\partial u_{n} / \partial x_{i}\right|>\alpha,\left|u_{n}\right| \leq \gamma\right\}}\left(\frac{1}{\alpha}\left|\frac{\partial u_{n}}{\partial x_{i}}\right|\right)^{p_{i}^{-}} d x+\lambda_{\left|u_{n}\right|}(\gamma) \\
\leq & \frac{1}{\alpha^{p_{i}^{-}}} \int_{\left\{\left|u_{n}\right| \leq \gamma\right\}}\left|\frac{\partial u_{n}}{\partial x_{i}}\right|^{p_{i}^{-}} d x+\lambda_{\left|u_{n}\right|}(\gamma) .
\end{aligned}
$$

Using (135) and (i), we get

$$
\lambda_{\left|\partial u_{n} / \partial x_{i}\right|}(\alpha) \leq C\left(\frac{\gamma}{\alpha^{p_{i}^{-}}}+\gamma^{-\widetilde{p}}\right)
$$

from which we deduce (ii).

By lemmas 3 and 23, it follows that $\left(u_{n}\right)_{n \in \mathbb{N}^{*}}$ is uniformly bounded in $L^{s_{0}}(\Omega)$ for some $1 \leq s_{0}<\widetilde{p}$, and in the same way, $\left(\left|\partial u_{n} / \partial x_{i}\right|\right)_{n \in \mathbb{N}^{*}}$ is uniformly bounded in $L^{s_{i}}(\Omega)$ for some $1 \leq s_{i}<\widetilde{p}_{i}$. From this, we get that the sequence $\left(u_{n}\right)_{n \in \mathbb{N}^{*}}$ is uniformly bounded in $W^{1, s}(\Omega)$, where $s=\min \left(s_{0}, s_{1}, \ldots, s_{N}\right)$. Consequently, we can extract a subsequence, still denoted $\left(u_{n}\right)$ satisfying

$$
\begin{gathered}
u_{n} \longrightarrow u \quad \text { a.e. in } \Omega, \text { in } L^{s}(\Omega), \\
u_{n} \rightarrow u \quad \text { in } W^{1, s}(\Omega), \\
\left|\frac{\partial u_{n}}{\partial x_{i}}-\frac{\partial u}{\partial x_{i}}\right| \rightarrow \mathscr{H}_{i}(x) \quad \text { in } L^{s}(\Omega), \forall i=1, \ldots, N .
\end{gathered}
$$

By the same way as in the proof of [16, Lemma 3.5] (see also [27]), we prove that

$$
\mathscr{H}_{i}(x)=0 \quad \text { a.e. } x \in \Omega \forall i=1, \ldots, N \text {. }
$$

We deduce from (139) that

$$
\begin{aligned}
& a_{i}\left(x, \frac{\partial u_{n}}{\partial x_{i}}\right) \\
& \quad \longrightarrow a_{i}\left(x, \frac{\partial u}{\partial x_{i}}\right) \text { a.e. in } \Omega, \text { in } L^{1}(\Omega), \forall i=1, \ldots, N .
\end{aligned}
$$

In order to pass to the limit in relation (134), we need also the following convergence results which can be proved by the same way as in [1]. 
Proposition 24. Assume (4)-(8), $f \in L^{1}(\Omega)$ and (132). Let $u_{n} \in W^{1, \vec{p}^{(\cdot)}}(\Omega)$ be the solution of (133). The sequence $\left(u_{n}\right)_{n \in \mathbb{N}}$ is Cauchy in measure. In particular, there exists a measurable function $u$ and a subsequence still denoted by $u_{n}$ such that $u_{n} \rightarrow u$ in measure.

Proposition 25. Assume (4)-(8), $f \in L^{1}(\Omega)$ and (132). Let $u_{n} \in W^{1, \vec{p}^{(\cdot)}}(\Omega)$ be the solution of (133). The following assertions hold.

(i) For all $i=1, \ldots, N, \partial u_{n} / \partial x_{i}$ converges in measure to the weak partial gradient of $u$.

(ii) For all $i=1, \ldots, N$ and all $k>0, a_{i}\left(x, \partial T_{k}\left(u_{n}\right) / \partial x_{i}\right)$ converges to $a_{i}\left(x, \partial T_{k}\left(u_{n}\right) / \partial x_{i}\right)$ in $L^{1}(\Omega)$ strongly and in $L^{p_{i}^{\prime}(\cdot)}(\Omega)$ weakly.

We can now pass to the limit in (134). To this end, let $\varphi \epsilon$ $W^{1, \vec{p}^{(\cdot)}}(\Omega) \cap L^{\infty}(\Omega)$. For any $k>0$, choose $T_{k}\left(u_{n}-\varphi\right)$ as a test function in (134), we get

$$
\begin{gathered}
\sum_{i=1}^{N} \int_{\Omega} a_{i}\left(x, \frac{\partial u_{n}}{\partial x_{i}}\right) \frac{\partial}{\partial x_{i}} T_{k}\left(u_{n}-\varphi\right) d x \\
+\int_{\Omega}\left|u_{n}\right|^{p_{M}(x)-2} u_{n} T_{k}\left(u_{n}-\varphi\right) d x \\
=\int_{\Omega} f_{n}(x) T_{k}\left(u_{n}-\varphi\right) d x .
\end{gathered}
$$

For the right-hand side of (141), the convergence is obvious since $f_{n}$ converges strongly to $f$ in $L^{1}(\Omega)$, and $T_{k}\left(u_{n}-\varphi\right)$ converges weakly-* to $T_{k}(u-\varphi)$ in $L^{\infty}(\Omega)$ and a.e in $\Omega$.

For the second term of (141), we have

$$
\begin{aligned}
& \int_{\Omega}\left|u_{n}\right|^{p_{M}(x)-2} u_{n} T_{k}\left(u_{n}-\varphi\right) d x \\
& =\int_{\Omega}\left(\left|u_{n}\right|^{p_{M}(x)-2} u_{n}-|\varphi|^{p_{M}(x)-2} \varphi\right) T_{k}\left(u_{n}-\varphi\right) d x \\
& \quad+\int_{\Omega}|\varphi|^{p_{M}(x)-2} \varphi T_{k}\left(u_{n}-\varphi\right) d x .
\end{aligned}
$$

The quantity $\left(\left|u_{n}\right|^{p_{M}(x)-2} u_{n}-|\varphi|^{p_{M}(x)-2} \varphi\right) T_{k}\left(u_{n}-\varphi\right)$ is nonnegative and since for all $x \in \Omega, s \mapsto \mid s^{p_{M}(x)-2} s$ is continuous; we get

$$
\begin{aligned}
& \left(\left|u_{n}\right|^{p_{M}(x)-2} u_{n}-|\varphi|^{p_{M}(x)-2} \varphi\right) T_{k}\left(u_{n}-\varphi\right) \\
& \quad \longrightarrow\left(|u|^{p_{M}(x)-2} u-|\varphi|^{p_{M}(x)-2} \varphi\right) T_{k}(u-\varphi) \quad \text { a.e. in } \Omega
\end{aligned}
$$

Then, it follows by Fatou's Lemma that

$$
\begin{gathered}
\liminf _{n \rightarrow+\infty} \int_{\Omega}\left(\left|u_{n}\right|^{p_{M}(x)-2} u_{n}-|\varphi|^{p_{M}(x)-2} \varphi\right) T_{k}\left(u_{n}-\varphi\right) d x \\
\geq \int_{\Omega}\left(|u|^{p_{M}(x)-2} u-|\varphi|^{p_{M}(x)-2} \varphi\right) T_{k}(u-\varphi) d x .
\end{gathered}
$$

Let us show that $|\varphi|^{p_{M}(x)-2} \varphi \in L^{1}(\Omega)$.

We have

$$
\begin{aligned}
\left.\int_{\Omega}|| \varphi\right|^{p_{M}(x)-2} \varphi \mid d x & =\int_{\Omega}|\varphi|^{p_{M}(x)-1} d x \\
& \leq \int_{\Omega}\left(\|\varphi\|_{\infty}\right)^{p_{M}(x)-1} d x .
\end{aligned}
$$

If $\|\varphi\|_{\infty} \leq 1$, then $\int_{\Omega} \|\left.\varphi\right|^{p_{M}(x)-2} \varphi \mid d x \leq \operatorname{meas}(\Omega)<+\infty$.

If $\|\varphi\|_{\infty}>1$, then

$$
\begin{aligned}
\left.\int_{\Omega}|| \varphi\right|^{p_{M}(x)-2} \varphi \mid d x & \leq \int_{\Omega}\left(\|\varphi\|_{\infty}\right)^{p_{M}^{+}-1} d x \\
& =\left(\|\varphi\|_{\infty}\right)^{p_{M}^{+}-1} \operatorname{meas}(\Omega)<+\infty .
\end{aligned}
$$

Hence, $|\varphi|^{p_{M}(x)-2} \varphi \in L^{1}(\Omega)$.

Since $T_{k}\left(u_{n}-\varphi\right)$ converges weakly-* to $T_{k}(u-\varphi)$ in $L^{\infty}(\Omega)$ and $|\varphi|^{p_{M}(x)-2} \varphi \in L^{1}(\Omega)$, it follows that

$$
\begin{array}{r}
\lim _{n \rightarrow+\infty} \int_{\Omega}|\varphi|^{p_{M}(x)-2} \varphi T_{k}\left(u_{n}-\varphi\right) d x \\
=\int_{\Omega}|\varphi|^{p_{M}(x)-2} \varphi T_{k}(u-\varphi) d x .
\end{array}
$$

For the first term of (141), we write it as follows:

$$
\begin{aligned}
\sum_{i=1}^{N} \int_{\left\{\left|u_{n}-\varphi\right| \leq k\right\}} a_{i}\left(x, \frac{\partial u_{n}}{\partial x_{i}}\right) \frac{\partial}{\partial x_{i}} u_{n} d x \\
-\sum_{i=1}^{N} \int_{\left\{\left|u_{n}-\varphi\right| \leq k\right\}} a_{i}\left(x, \frac{\partial u_{n}}{\partial x_{i}}\right) \frac{\partial}{\partial x_{i}} \varphi d x .
\end{aligned}
$$

The first term of (148) is nonnegative by (7), then by Fatou's Lemma and (138), we get

$$
\begin{aligned}
& \sum_{i=1}^{N} \int_{\{|u-\varphi| \leq k\}} a_{i}\left(x, \frac{\partial u}{\partial x_{i}}\right) \frac{\partial}{\partial x_{i}} u d x \\
& \quad \leq \liminf _{n \rightarrow \infty} \sum_{i=1}^{N} \int_{\left\{\left|u_{n}-\varphi\right| \leq k\right\}} a_{i}\left(x, \frac{\partial u_{n}}{\partial x_{i}}\right) \frac{\partial}{\partial x_{i}} u_{n} d x .
\end{aligned}
$$

According to Proposition 25, the second term of (148) converges to

$$
\sum_{i=1}^{N} \int_{\{|u-\varphi| \leq k\}} a_{i}\left(x, \frac{\partial u}{\partial x_{i}}\right) \frac{\partial}{\partial x_{i}} \varphi d x .
$$

Combining the previous convergence results, we obtain

$$
\begin{gathered}
\sum_{i=1}^{N} \int_{\Omega} a_{i}\left(x, \frac{\partial u}{\partial x_{i}}\right) \frac{\partial}{\partial x_{i}} T_{k}(u-\varphi) d x \\
\quad+\int_{\Omega}|u|^{p_{M}(x)-2} u T_{k}(u-\varphi) d x \\
\leq \int_{\Omega} f(x) T_{k}(u-\varphi) d x .
\end{gathered}
$$




\section{References}

[1] B. K. Bonzi and S. Ouaro, "Entropy solutions for a doubly nonlinear elliptic problem with variable exponent," Journal of Mathematical Analysis and Applications, vol. 370, no. 2, pp. 392405, 2010.

[2] M.-M. Boureanu and V. D. Rădulescu, "Anisotropic Neumann problems in Sobolev spaces with variable exponent," Nonlinear Analysis: Theory, Methods \& Applications, vol. 75, no. 12, pp. 4471-4482, 2012.

[3] Y. Chen, S. Levine, and M. Rao, "Variable exponent, linear growth functionals in image restoration," SIAM Journal on Applied Mathematics, vol. 66, no. 4, pp. 1383-1406, 2006.

[4] L. Diening, Theoretical and numerical results for electrorheological fluids [Ph.D. thesis], University of Frieburg, Freiburg, Germany, 2002.

[5] L. Diening, "Riesz potential and Sobolev embeddings on generalized Lebesgue and Sobolev spaces $L^{p(.)}$ and $W^{1, p(.) \text {," }}$ Mathematische Nachrichten, vol. 268, pp. 31-43, 2004.

[6] D. E. Edmunds and J. Rákosník, "Density of smooth functions in $W^{k, p(x)}(\Omega)$," Proceedings of the Royal Society of London. Series A, vol. 437, no. 1899, pp. 229-236, 1992.

[7] D. E. Edmunds and J. Rákosník, "Sobolev embeddings with variable exponent," Studia Mathematica, vol. 143, no. 3, pp. 267293, 2000

[8] D. E. Edmunds and J. Rákosník, "Sobolev embeddings with variable exponent. II," Mathematische Nachrichten, vol. 246/247, pp. 53-67, 2002.

[9] A. El Hamidi, "Existence results to elliptic systems with nonstandard growth conditions," Journal of Mathematical Analysis and Applications, vol. 300, no. 1, pp. 30-42, 2004.

[10] X. Fan and D. Zhao, "On the spaces $L^{p(.)}(\Omega)$ and $W^{1, p(.)}(\Omega)$," Journal of Mathematical Analysis and Applications, vol. 263, no. 2, pp. 424-446, 2001.

[11] X. Fan, "Anisotropic variable exponent Sobolev spaces and $\vec{p}()-$. Laplacian equations," Complex Variables and Elliptic Equations, vol. 55, pp. 1-20, 2010.

[12] X.-L. Fan and Q.-H. Zhang, "Existence of solutions for $p(x)$ Laplacian Dirichlet problem," Nonlinear Analysis: Theory, Methods \& Applications, vol. 52, no. 8, pp. 1843-1852, 2003.

[13] P. Harjulehto, P. Hästö, Út V. Lê, and M. Nuortio, "Overview of differential equations with non-standard growth," Nonlinear Analysis: Theory, Methods \& Applications, vol. 72, no. 12, pp. 4551-4574, 2010.

[14] P. Harjulehto, P. Hästö, M. Koskenoja, and S. Varonen, "The Dirichlet energy integral and variable exponent Sobolev spaces with zero boundary values," Potential Analysis, vol. 25, no. 3, pp. 205-222, 2006.

[15] B. Koné, S. Ouaro, and S. Traoré, "Weak solutions for anisotropic nonlinear elliptic equations with variable exponents," Electronic Journal of Differential Equations, vol. 2009, no. 144, pp. 1-11, 2009.

[16] B. Koné, S. Ouaro, and S. Soma, "Weak solutions for anisotropic nonlinear elliptic problem with variable exponent and measure data," International Journal of Evolution Equations, vol. 5, no. 3, p. 327-350 (2011), 2010.

[17] O. Kováčik and J. Rákosník, "On spaces $L^{p(x)}$ and $W^{1, p(x), "}$ Czechoslovak Mathematical Journal, vol. 41(116), no. 4, pp. 592618, 1991.

[18] M. Mihăilescu, P. Pucci, and V. Rădulescu, "Eigenvalue problems for anisotropic quasilinear elliptic equations with variable exponent," Journal of Mathematical Analysis and Applications, vol. 340, no. 1, pp. 687-698, 2008.
[19] M. Mihăilescu and V. Rădulescu, "A multiplicity result for a nonlinear degenerate problem arising in the theory of electrorheological fluids," Proceedings of the Royal Society of London. Series A, vol. 462, no. 2073, pp. 2625-2641, 2006.

[20] S. Ouaro, "Well-posedness results for anisotropic nonlinear elliptic equations with variable exponent and $L^{1}$-data," Cubo, vol. 12, no. 1, pp. 133-148, 2010.

[21] K. R. Rajagopal and M. Ruzicka;, "Mathematical modelling of electrorheological fluids," Continuum Mechanics and Thermodynamics, vol. 13, pp. 59-78, 2001.

[22] M. Ruzicka, Electrorheological Fluids: Modeling and Mathematical Theory, vol. 1748 of Lecture Notes in Mathematics, Springer, Berlin, Germany, 2000.

[23] M. Sanchón and J. M. Urbano, "Entropy solutions for the $p(x)$ Laplace equation," Transactions of the American Mathematical Society, vol. 361, no. 12, pp. 6387-6405, 2009.

[24] D. Stancu-Dumitru, "Multiplicity of solutions for anisotropic quasilinear elliptic equations with variable exponents," Bulletin of the Belgian Mathematical Society, vol. 17, no. 5, pp. 875-889, 2010.

[25] D. Stancu-Dumitru, "Two nontrivial solutions for a class of anisotropic variable exponent problems," Taiwanese Journal of Mathematics, vol. 16, no. 4, pp. 1205-1219, 2012.

[26] D. Stancu-Dumitru, "Multiplicity of solutions for a nonlinear degenerate problem in anisotropic variable exponent spaces," Bulletin of the Malaysian Mathematical Sciences Society, vol. 36, no. 21, pp. 117-130, 2013.

[27] M. Bendahmane and K. H. Karlsen, "Anisotropic nonlinear elliptic systems with measure data and anisotropic harmonic maps into spheres," Electronic Journal of Differential Equations, vol. 2006, no. 46, pp. 1-30, 2006.

[28] P. Benilan, H. Brezis, and M. G. Crandall, "A semilinear equation in $L^{1}(\mathbb{R})^{N}$," Annali della Scuola Normale Superiore di Pisa, vol. 2, no. 4, pp. 523-555, 1975.

[29] L. Diening, P. Harjulehto, P. Hästö, and M. Ruzicka, Lebesgue and Sobolev Spaces with Variable Exponents, vol. 2017 of Lecture Notes in Mathematics, Springer, Heidelberg, Germany, 2011.

[30] M. Troisi, “Teoremi di inclusione per spazi di Sobolev non isotropi," Ricerche di Matematica, vol. 18, pp. 3-24, 1969.

[31] A. J. Kurdila and M. Zabarankin, Convex Functional Analysis, Systems \& Control: Foundations \& Applications, Birkhäuser, Basel, Switzerland, 2005.

[32] M. Struwe, Variational Methods: Applications to Nonlinear Partial Dierential Equations and Hamiltonian Systems, vol. 34 of Results in Mathematics and Related Areas (3), Springer, Berlin, Germany, 2nd edition, 1996.

[33] H. Brezis, Analyse Fonctionnelle: Théorie et Applications, Collection Mathématiques Appliquées pour la Maîtrise, Masson, Paris, Farnce, 1983.

[34] A. Alvino, L. Boccardo, V. Ferone, L. Orsina, and G. Trombetti, "Existence results for nonlinear elliptic equations with degenerate coercivity," Annali di Matematica Pura ed Applicata, vol. 182, no. 1, pp. 53-79, 2003.

[35] P. Bénilan, L. Boccardo, T. Gallouët, R. Gariepy, M. Pierre, and J. L. Vázquez, "An $L^{1}$-theory of existence and uniqueness of solutions of nonlinear elliptic equations," Annali della Scuola Normale Superiore di Pisa, vol. 22, no. 2, pp. 241-273, 1995. 


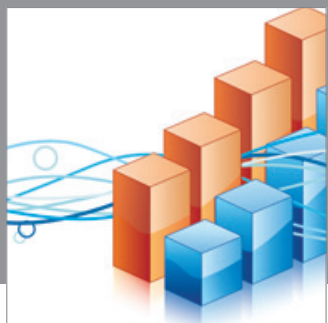

Advances in

Operations Research

mansans

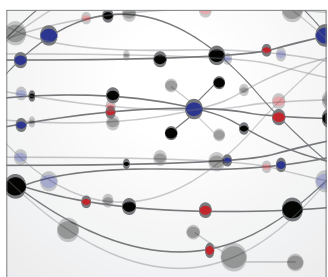

The Scientific World Journal
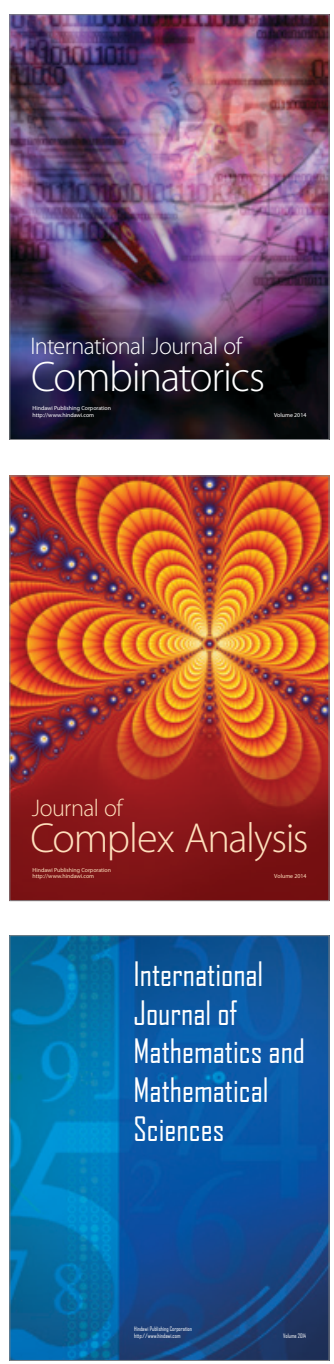
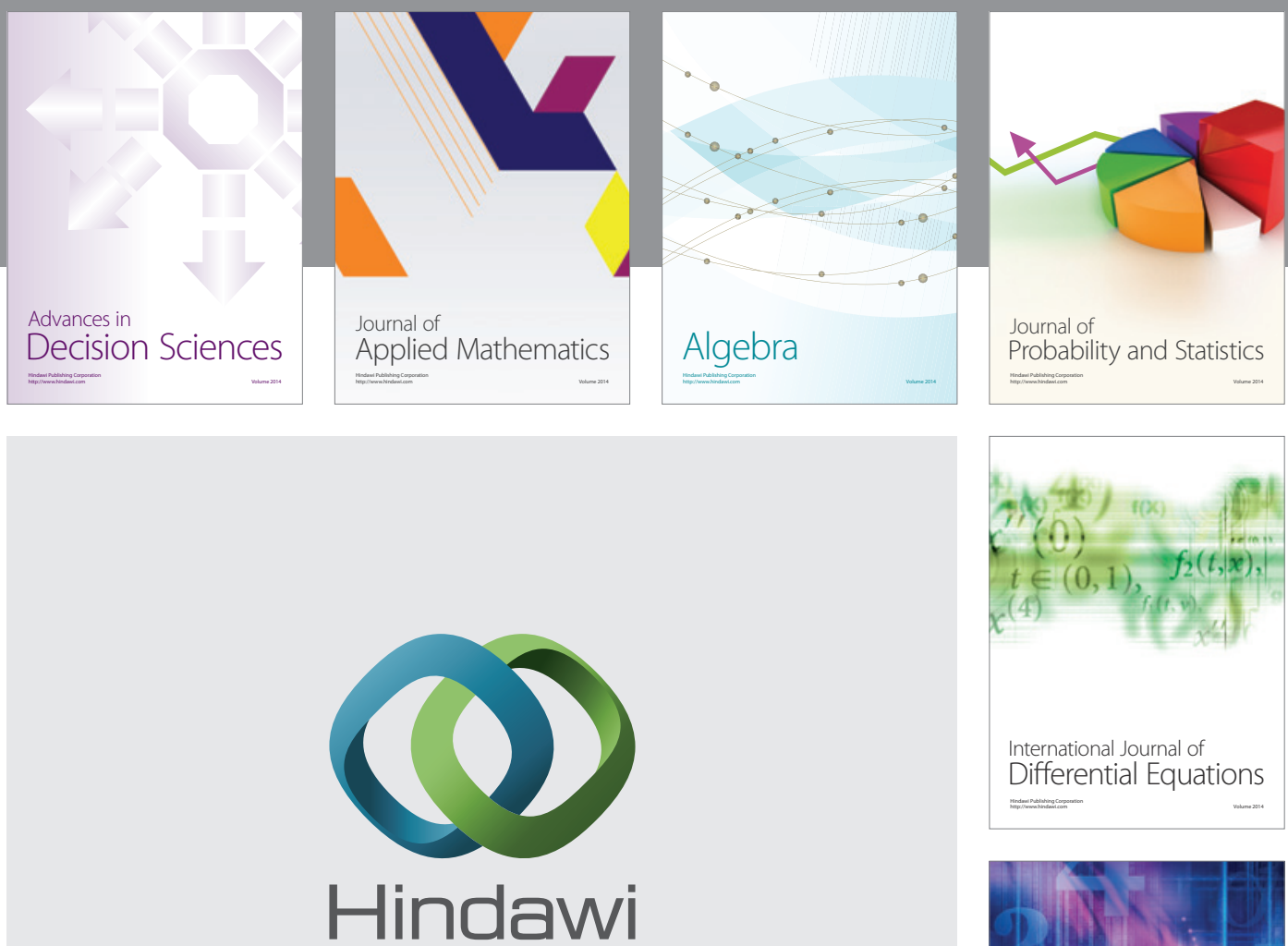

Submit your manuscripts at http://www.hindawi.com
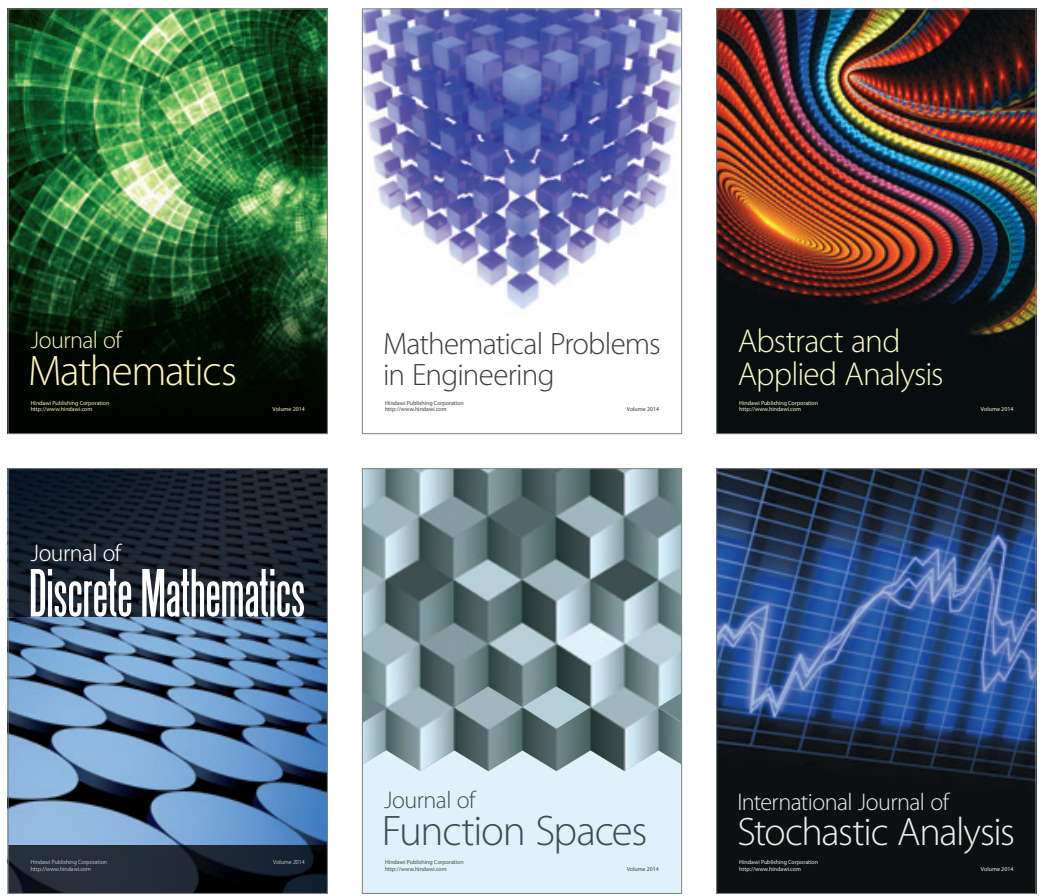

Journal of

Function Spaces

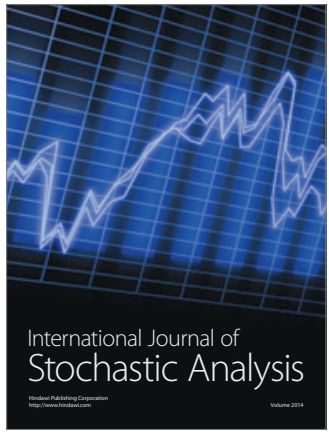

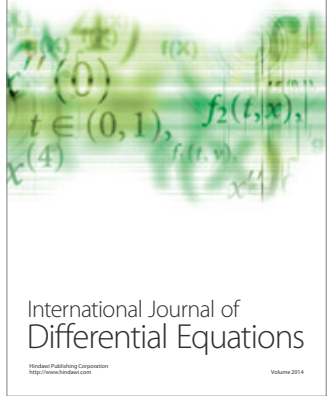
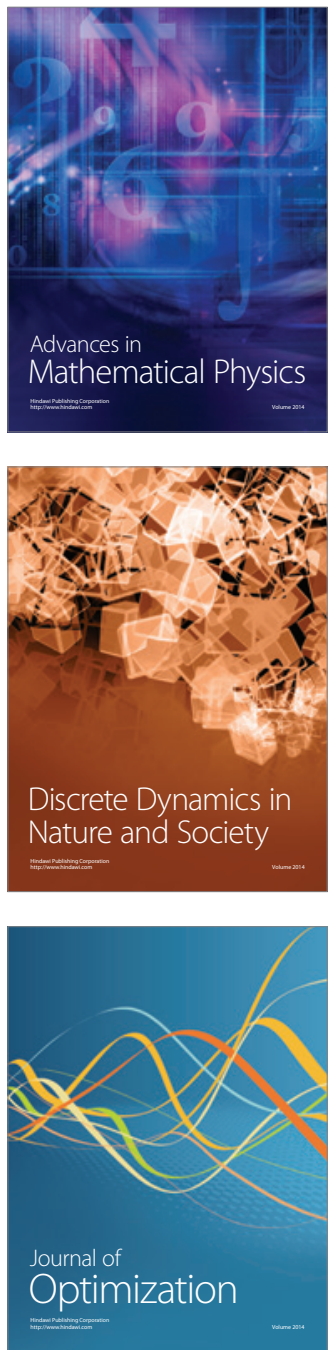